\title{
ONE-PARAMETER FAMILIES AND NETS OF PLANE CURVES*
}

BY

\section{E. J. WILCZYNSKI}

\section{Introduction.}

In most treatments of the theory of one-parameter families of plane curves attention is concentrated altogether upon the discussion of the envelope. More recently, some authors, especially Lilienthal $\dagger$ and Scheffers, $\ddagger$ have considered some other problems associated with such families of curves, but all of these are of a metric nature. In the present paper, the point of view is that of projective differential geometry and the analytic treatment is based upon the invariant theory of a certain completely integrable system of partial differential equations of the second order. Incidentally a simple new geometrical interpretation is found for the well-known Laplacian transformation of a linear partial differential equation of the second order, which enables one to make use of the results of that theory for the purpose of studying nets of plane curves. The osculating conics of the curves of the net are also determined and their properties investigated for the first time.

\section{$\S 1$. The differential equations of the problem. Integrability conditions.}

Let the homogeneous coördinates $y^{(k)}$ of a point $P_{y}$ of the plane be given as analytic functions of two independent variables;

$$
y^{(k)}=f^{(k)}(u, v) \quad(k=1,2,3) .
$$

Clearly the equations $u=$ const. and $v=$ const. will, in general, give rise to two one-parameter families of plane curves, such that, if we consider merely the portions of these curves in a certain domain, one and only one curve of each family will pass through every point of the domain. We shall speak of these two families together as a net, so that equations (1) define a net. This net will degenerate if and only if the ratios $y^{(1)}: y^{(2)}: y^{(3)}$ can be expressed as functions of a single independent variable, i. e., if and only if there exist three non-vanishing

* Presented to the Society (Chicago) December 29, 1910.

† LiLI Enthal, Grundlage einer Krümmungslehre der Kurvenscharen, Leipzig, 1896; Vorlesungen über Differentialgeometrie, Leipzig, 1908.

† Sch EFFERS, Kgl. Sächsische. Gesellschaft der Wissenschaften, Berichte, vol. 57 (1905); Mathematische Annalen, vol. 60 (1905). 
functions $\alpha, \beta, \gamma$ of $u$ and $v$ such that

$$
\alpha y_{u}^{(k)}+\beta y_{o}^{(k)}+\gamma y^{(k)}=0
$$

$(k=1,2,3)$.

Consequently, the net defined by equations (1) is degenerate if and only if the determinant

vanishes identically.

$$
D=\left|\begin{array}{lll}
y_{u}^{(1)}, & y_{u}^{(2)}, & y_{u}^{(3)} \\
y_{v}^{(1)}, & y_{v}^{(2)}, & y_{v}^{(3)} \\
y^{(1)}, & y^{(2)}, & y^{(3)}
\end{array}\right|
$$

In particular, in order that the net may not degenerate, $y^{(1)}, y^{(2)}, y^{(3)}$ must be linearly independent.

Let us assume that equations (1) define a non-degenerate net. Then $D$ does not vanish, and we can always find a system of partial differential equations of the form

$$
\begin{aligned}
& y_{u u}=a y_{u}+b y_{v}+c y, \\
& y_{u v}=a^{\prime} y_{u}+b^{\prime} y_{v}+c^{\prime} y, \\
& y_{v v}=a^{\prime \prime} y_{u}+b^{\prime \prime} y_{v}+c^{\prime \prime} y,
\end{aligned}
$$

satisfied by the three functions $y^{(1)}, y^{(2)}, y^{(3)}$.

It is easy to show that, conversely, a system of form (4) with analytic coefficients has just three linearly independent analytic solutions $y^{(1)}, y^{(2)}, y^{(3)}$, for which the determinant $D$ does not vanish identically, provided that the integrability conditions

$$
\frac{\partial y_{u u}}{\partial v}=\frac{\partial y_{u v}}{\partial u}, \quad \frac{\partial y_{u v}}{\partial u}=\frac{\partial y_{v v}}{\partial u}
$$

are satisfied identically, i. e., without the addition of one or more equations of the form (2) to system (4). The identical validity of these integrability conditions implies the following six equations :

$$
\begin{aligned}
b a^{\prime \prime}+a_{v} & =a^{\prime} b^{\prime}+c^{\prime}+a_{u}^{\prime}, \\
a b^{\prime}+b b^{\prime \prime}+c+b_{v} & =a^{\prime} b+b^{\prime 2}+b_{u}^{\prime}, \\
a c^{\prime}+b c^{\prime \prime}+c_{v} & =a^{\prime} c+b^{\prime} c^{\prime}+c_{u}^{\prime}, \\
a^{\prime 2}+b^{\prime} a^{\prime \prime}+a_{v}^{\prime} & =a a^{\prime \prime}+a^{\prime} b^{\prime \prime}+c^{\prime \prime}+a_{u}^{\prime \prime}, \\
a^{\prime} b^{\prime}+c^{\prime}+b_{v}^{\prime} & =a^{\prime \prime} b+b_{u}^{\prime \prime}, \\
a^{\prime} c^{\prime}+b^{\prime} c^{\prime \prime}+c_{v}^{\prime} & =a^{\prime \prime} c+b^{\prime \prime} c^{\prime}+c_{u}^{\prime \prime} .
\end{aligned}
$$

Now every non-degenerate net of plane curves determines a unique system of form (4), for which the conditions (5) must of course be satisfied. On the other hand every system of form (4), for which the integrability conditions (5) are 
satisfied, has three linearly independent solutions $y^{(1)}, y^{(2)}, y^{(3)}$, for which the determinant $D$ does not vanish identically, and the most general solutions of such a system will be

$$
\bar{y}^{(k)}=c_{k 1} y^{(1)}+c_{k 2} y^{(2)}+c_{k 3} y^{(3)} \quad(k=1,2,3), \quad\left|c_{k l}\right| \neq 0 .
$$

Consequently, if we interpret $y^{(1)}, y^{(2)}, y^{(3)}$ as the homogeneous coördinates of a point in a plane, any fundamental system of solutions of (4) defines a non-degenerate net of plane curves, and the net defined by the most general fundamental system of solutions of (4) will be a projective transformation of this.

We see, therefore, that all projective differential properties of a net must be expressible in terms of the coefficients and variables of the system of partial differential equations (4).

It is possible to simplify the integrability conditions (5) somewhat. From the first and fifth of these we easily deduce

so that we may write

$$
a_{v}+b_{v}^{\prime}=a_{u}^{\prime}+b_{u}^{\prime \prime},
$$

$$
a+b^{\prime}=f_{u}, \quad a^{\prime}+b^{\prime \prime}=f_{v},
$$

as a consequence of which (5) may be written as follows ;

$$
\begin{aligned}
f_{u v}-b_{v}^{\prime}-a_{u}^{\prime} & =c^{\prime}+a^{\prime} b^{\prime}-a^{\prime \prime} b, \\
b_{v}-b_{u}^{\prime} & =-b^{\prime} f_{u}-b f_{v}-c+2 b^{\prime 2}+2 a^{\prime} b, \\
c_{v}-c_{u}^{\prime} & =-c^{\prime} f_{u}+2 b^{\prime} c^{\prime}+a^{\prime} c-b c^{\prime \prime}, \\
a_{v}^{\prime}-a_{u}^{\prime \prime} & =a^{\prime \prime} f_{u}+a^{\prime} f_{v}+c^{\prime \prime}-2{a^{\prime}}^{2}-2 a^{\prime \prime} b^{\prime}, \\
c_{v}^{\prime}-c_{u}^{\prime \prime} & =c^{\prime} f_{v}-2 a^{\prime} c^{\prime}+a^{\prime \prime} c-b^{\prime} c^{\prime \prime} .
\end{aligned}
$$

$\S 2$. The seminvariants and the canonical form of the system.

The analytic representation of a net by a system of equations such as (1) is not unique. The coefficients of system (4) therefore do not depend merely upon the geometric properties of the net, but also upon the particular form of analytic representation adopted. In the first place, since $y^{(1)}, y^{(2)}, y^{(3)}$ are homogeneous coördinates, only those functions can have a geometrical significance which depend only upon the ratios of these quantities. Now, a transformation of the form

$$
y^{(k)}=\lambda(u, v) y^{(k)} \quad(k=1,2,3)
$$

leaves these ratios unaltered whatever value the arbitrary function $\lambda(u, v)$ may have. Consequently it will be necessary to find those combinations of the coefficients of (4) and of their derivatives, which are left unchanged by any transformation of the form

$$
y=\lambda(u, v) \bar{y}
$$


We shall call them seminvariants. The corresponding invariant functions which depend also upon $y, y_{u}, y_{v}$, shall be called semicovariants.

We proceed to carry out the transformation (8). We find immediately

$$
\begin{aligned}
y_{u} & =\lambda \bar{y}_{u}+\lambda_{u} \bar{y}, \quad y_{v}=\lambda \bar{y}_{v}+\lambda_{v} \bar{y}, \\
y_{u u} & =\lambda \bar{y}_{u u}+2 \lambda_{u} \bar{y}_{u}+\lambda_{u u} \bar{y} \\
y_{u v} & =\lambda \bar{y}_{u v}+\lambda_{v} \bar{y}_{u}+\lambda_{u} \bar{y}_{v}+\lambda_{u v} \bar{y}, \\
y_{v v} & =\lambda \bar{y}_{v v}+2 \lambda_{v} \bar{y}_{v}+\lambda_{v v} \bar{y} .
\end{aligned}
$$

If these values be substituted in system (4), and the new coefficients be denoted by $\bar{a}, \bar{b}$, etc., we shall find :

$$
\begin{aligned}
& \bar{a}=a-2 \frac{\lambda_{u}}{\lambda}, \quad \bar{b}=b, \quad \bar{c}=c+a \frac{\lambda_{u}}{\lambda}+b \frac{\lambda_{v}}{\lambda}-\frac{\lambda_{u u}}{\lambda}, \\
& \text { (10) } \bar{a}^{\prime}=a^{\prime}-\frac{\lambda_{v}}{\lambda}, \quad \bar{b}^{\prime}=b^{\prime}-\frac{\lambda_{u}}{\lambda}, \quad \bar{c}^{\prime}=c^{\prime}+a^{\prime} \frac{\lambda_{u}}{\lambda}+b^{\prime} \frac{\lambda_{v}}{\lambda}-\frac{\lambda_{u v}}{\lambda} \text {, } \\
& \bar{a}^{\prime \prime}=a^{\prime \prime}, \quad \quad \bar{b}^{\prime \prime}=b^{\prime \prime}-2 \frac{\lambda_{n}}{\lambda}, \quad \bar{c}^{\prime \prime}=c^{\prime \prime}+a^{\prime \prime} \frac{\lambda_{u}}{\lambda}+b^{\prime \prime} \frac{\lambda_{v}}{\lambda}-\frac{\lambda_{v v}}{\lambda} .
\end{aligned}
$$

According to (6) we have

If we write similarly

$$
a+b^{\prime}=f_{u}, \quad a^{\prime}+b^{\prime \prime}=f_{v} .
$$

we shall find

$$
\bar{a}+\bar{b}^{\prime}=\bar{f}_{u}, \quad \bar{a}^{\prime}+\bar{b}^{\prime \prime}=\bar{f}_{v},
$$

$$
\overline{f_{u}}=f_{u}-3 \frac{\lambda_{u}}{\lambda}, \quad \overline{f_{v}}=f_{v}-3 \frac{\lambda_{v}}{\lambda} .
$$

We may, therefore, transform (4) into another system of the same form for which

$$
\bar{a}+\bar{b}^{\prime}=0, \quad \bar{a}^{\prime}+\bar{b}^{\prime \prime}=0,
$$

if we choose $\lambda(u, v)$ in the transformation (8) subject to the conditions

$$
\frac{\lambda_{u}}{\lambda}=\frac{1}{3} f_{u}, \quad \frac{\lambda}{\lambda}=\frac{1}{3} f_{v}, \quad \lambda=\text { const. } e^{\frac{1}{3} f} .
$$

Let us actually make this particular transformation, and let us denote the coefficients of the resulting system by capital letters. We shall have

$$
\begin{array}{lll}
A=a-\frac{2}{3} f_{u}, & B=b, & C=c+\frac{1}{3} a f_{u}+\frac{1}{3} b f_{v}^{\prime}-\frac{1}{3} f_{u u}-\frac{1}{9} f_{u}^{2}, \\
A^{\prime}=a^{\prime}-\frac{1}{8} f_{v}, & B^{\prime}=b^{\prime}-\frac{1}{3} f_{u}, & C^{\prime}=c^{\prime}+\frac{1}{3} a^{\prime} f_{u}+\frac{1}{3} b^{\prime} f_{v}-\frac{1}{3} f_{u v}-\frac{1}{9} f_{u} f_{v}, \\
A^{\prime \prime}=a^{\prime \prime}, & B^{\prime \prime}=b^{\prime \prime}-\frac{2}{3} f_{v}, & C^{\prime \prime}=c^{\prime \prime}+\frac{1}{3} a^{\prime \prime} f_{u}+\frac{1}{3} b^{\prime \prime} f_{v}-\frac{1}{3} f_{v v}-\frac{1}{9} f_{v}^{2}, \\
& A+B^{\prime}=0, & A^{\prime}+B^{\prime \prime}=0 .
\end{array}
$$

An integrable system of form (4) can always be transformed into another 
one whose uniquely determined coefficients are given by equations (13), and satisfy the characteristic relations

$$
A+B^{\prime}=0, \quad A^{\prime}+B^{\prime \prime}=0 .
$$

The most general transformation which effects this reduction is

$$
y=\text { const. } \bar{y} e^{\frac{1}{3} f} \text {. }
$$

We shall say that, as a result of this transformation, the system has been reduced to its canonical form.

The coefficients $A, B, C, \ldots$ of a system in its canonical form are seminvariants of system (4). This follows at once from the uniqueness of the canonical form, and may also be verified for each of the quantities $A, B, C, \ldots$ by direct calculation. Now let $f\left(a, b, \ldots ; a_{u}, b_{u}, \ldots\right)$ be any seminvariant depending not merely upon the coefficients of (4) but also upon their derivatives to any order. Since a seminvariant does not change its value if $a, b, c, \ldots$ in its expression be replaced by $\bar{a}, \bar{b}, \bar{c}, \ldots$, the coefficients of any system obtained from (4) by a transformation of the form $y=\lambda \bar{y}$, we shall have in particular

$$
f\left(a, b, c, \ldots ; a_{u}, b_{u}, \cdots\right)=f\left(A, B, C, \ldots ; A_{u}, B_{u}, \cdots\right) ;
$$

i. e., it is a function of $A, B, C, \ldots$ and of their derivatives. Conversely, it is evident that any function of $A, B, C, \ldots$ and of their derivatives is a seminvariant.

Consequently, every seminvariant of system (4) is a function of the seven fundamental ones, $B, C, A^{\prime}, B^{\prime}, C^{\prime}, A^{\prime \prime}, C^{\prime \prime}$, and of the various derivatives of these quantities. And every function of these variables is a seminvariant.

Since, by means of the equations (4), any function of $y, y_{u}, y_{v}$, and the higher derivatives of $y$ may be reduced to one of $y, y_{u}$ and $y_{v}$ alone, we may confine our search for semi-covariants to those depending upon these three variables only. Of course $y$ itself is a relative semi-covariant. It is easy to verify that

$$
\rho=y_{u}-b^{\prime} y, \quad \sigma=y_{v}-a^{\prime} y
$$

are also semi-covariants. Any relative semi-covariant may be expressed as a homogeneous function of these three and of seminvariants.*

* It is easy to show that an absolute semi-covariant must be a homogeneous function of degree zero of $y, y_{u}$ and $y_{v}$. Any such function $C$ may be written as a funotion of the two ratios $\rho / y$, $\sigma / y$ and of the coefficients of (4);

$$
C=f\left(\frac{\rho}{y}, \frac{\sigma}{y}, a, b, c, \cdots\right)
$$

But, as in the argument for seminvariants, this reduces to

$$
C=f\left(\frac{\rho}{y}, \frac{\sigma}{y}, A, B, C, \cdots\right)
$$

whence follows easily the theorem as stated. 
The integrability conditions of system (4) must, of course, be left invariant by the transformation $y=\lambda \bar{y}$; i. e., they must be capable of expression in terms of seminvariants. We obtain them by writing the conditions that the system in its canonical form must be integrable. This gives the integrability conditions of (4) in the form:

$$
\begin{aligned}
& B_{v}^{\prime}+A_{u}^{\prime}+C^{\prime}+A^{\prime} B^{\prime}-A^{\prime \prime} B=0, \\
& B_{v}-B_{u}^{\prime}=-C+2{B^{\prime}}^{2}+2 A^{\prime} B, \\
& C_{v}-C_{u}^{\prime}=2 B^{\prime} C^{\prime}+A^{\prime} C-B C^{\prime \prime}, \\
& A_{v}^{\prime}-A_{u}^{\prime \prime}=C^{\prime \prime}-2 A^{\prime 2}-2 A^{\prime \prime} B^{\prime}, \\
& C_{v}^{\prime}-C_{u}^{\prime \prime}=-2 A^{\prime} C^{\prime}+A^{\prime \prime} C-B^{\prime} C^{\prime \prime} .
\end{aligned}
$$

\section{§3. The invariants.}

We have not yet found an adequate analytic equivalent for the geometric properties of the net. To be sure, the seminvariants depend merely upon the ratios of $y^{(1)}, y^{(2)}, y^{(3)}$, and not upon their absolute values; but they also depend upon the parametric representation which has been adopted for the net. Clearly the net remains unchanged by any transformation of the form

$$
\bar{u}=U(u), \quad \bar{v}=V(v),
$$

where $U$ and $V$ are arbitrary functions of the single variables indicated, while any more general transformation of these variables would change the net. We must therefore find those combinations of the seminvariants and semi-covariants which remain unaltered as a result of a transformation of form (15). We shall call them invariants and covariants respectively. These functions are intrinsically connected with the projective differential properties of the net. Strictly speaking we ought to adjoin the further transformation

$$
\bar{u}=v, \quad \bar{v}=u,
$$

which merely interchanges the two one-parameter families of the net, but we shall prefer to think of these two families separately, so that formally we are constructing a theory of the two component one-parameter families rather than a theory of the net itself. From our invariants it is very easy to construct those invariants which may in all strictness be called invariants of the net. They are, of course, symmetric combinations of the invariants considered here.

Let us transform system (4) by equations (15). We find

$$
\frac{\partial y}{\partial u}=\frac{\partial y}{\partial \bar{u}} U^{\prime}, \quad \frac{\partial y}{\partial v}=\frac{\partial y}{\partial \bar{v}} V^{\prime},
$$




$$
\begin{aligned}
\frac{\partial^{2} y}{\partial u^{2}} & =\frac{\partial^{2} y}{\partial \bar{u}^{2}} U^{\prime 2}+\frac{\partial y}{\partial \bar{u}} U^{\prime \prime} \\
\frac{\partial^{2} y}{\partial u \partial v} & =\frac{\partial^{2} y}{\partial \bar{u} \partial \bar{v}} U^{\prime} V^{\prime} \\
\frac{\partial^{2} y}{\partial v^{2}} & =\frac{\partial^{2} y}{\partial \bar{v}^{2}} V^{\prime 2}+\frac{\partial y}{\partial \bar{v}} V^{\prime \prime}
\end{aligned}
$$

where the accents indicate differentiation in the customary way. If we substitute these values into system (4) and denote the coefficients of the new system by $\bar{a}, \bar{b}$, etc., we shall find :

$$
\begin{aligned}
& \bar{a}=\frac{1}{U^{\prime}}(a-\eta), \quad \bar{b}=\frac{V^{\prime} b}{U^{\prime 2}}, \quad \bar{c}=\frac{c}{U^{\prime 2}}, \\
& \bar{a}^{\prime}=\frac{a^{\prime}}{V^{\prime}}, \quad \bar{b}^{\prime}=\frac{b^{\prime}}{U^{\prime}}, \quad \bar{c}^{\prime}=\frac{c^{\prime}}{U^{\prime} V^{\prime}}, \\
& \bar{a}^{\prime \prime}=\frac{U^{\prime} a^{\prime \prime}}{V^{\prime 2}} \quad \bar{b}^{\prime \prime}=\frac{1}{V^{\prime}}\left(b^{\prime \prime}-\zeta\right), \quad \bar{c}^{\prime \prime}=\frac{c^{\prime \prime}}{V^{\prime 2}},
\end{aligned}
$$

where

$$
\eta=\frac{U^{\prime \prime}}{U^{\prime}}, \quad \zeta=\frac{V^{\prime \prime}}{V^{\prime}}
$$

so that $\eta$ is a function of $u$, and $\zeta$ of $v$ only. We find further

$$
\begin{aligned}
& \bar{f}_{u}=\frac{1}{U^{\prime}}\left(f_{u}-\eta\right), \quad \bar{f}_{v}=\frac{1}{V^{\prime}}\left(f_{v}-\zeta\right), \\
& \bar{f}_{u u}=\frac{1}{\left(U^{\prime}\right)^{2}}\left(f_{u u}-\eta f_{u}+\eta^{2}-\eta_{u}\right), \\
& \bar{f}_{u v}=\frac{1}{U^{\prime} V^{\prime}} f_{u v}, \\
& \bar{f}_{v v}=\frac{1}{\left(V^{\prime}\right)^{2}}\left(f_{v v}-\zeta f_{v}+\zeta^{2}-\zeta_{v}\right),
\end{aligned}
$$

where the notations $\bar{f}_{u}, \bar{f}_{u u}$, etc., are abbreviations for $\partial \bar{f} / \partial \bar{u}, \partial^{2} \bar{f} / \partial \bar{u}^{2}$, etc.

If now we make use of the expressions (13) of the fundamental seminvariants, and denote by $\bar{A}, \bar{B}$, etc., the corresponding seminvariants of the transformed system of differential equations, we shall find

$$
\bar{B}=\frac{V^{\prime}}{\left(U^{\prime}\right)^{2}} B, \quad \bar{C}=\frac{1}{\left(U^{\prime}\right)^{2}}\left(C-\frac{1}{3} A \eta-\frac{1}{3} B \zeta-\frac{1}{9} \eta^{2}+\frac{1}{3} \eta_{u}\right),
$$




$$
\bar{A}^{\prime}=\frac{1}{V^{\prime}}\left(A^{\prime}+\frac{1}{3} \zeta\right), \quad \bar{B}^{\prime}=\frac{1}{U^{\prime}}\left(B^{\prime}+\frac{1}{3} \eta\right)
$$

$$
\begin{aligned}
& \bar{C}^{\prime}=\frac{1}{U^{\prime} V^{\prime}}\left(C^{\prime}-\frac{1}{3} A^{\prime} \eta-\frac{1}{3} B^{\prime} \zeta-\frac{1}{9} \eta \zeta\right), \\
& \bar{A}^{\prime \prime}=\frac{U^{\prime}}{\left(V^{\prime}\right)^{2}} A^{\prime \prime}, \quad \bar{C}^{\prime \prime}=\frac{1}{\left(V^{\prime}\right)^{2}}\left(C^{\prime \prime}-\frac{1}{3} A^{\prime \prime} \eta-\frac{1}{3} B^{\prime \prime} \zeta-\frac{1}{9} \zeta^{2}+\frac{1}{3} \zeta_{v}\right) .
\end{aligned}
$$

We see that the seminvariants $B$ and $A^{\prime \prime}$ are at the same time (relative) invariants, and it is easy to verify the invariance of all of the following quantities :

$$
\begin{aligned}
\mathfrak{B} & =B, & \mathfrak{E} & =C-B_{u}^{\prime}-2{B^{\prime 2}}^{2}+A^{\prime} B, \\
\mathfrak{U}^{\prime} & =A^{\prime}+\frac{1}{6} \frac{A_{v}^{\prime \prime}}{A^{\prime \prime}}, & \mathfrak{B}^{\prime} & =B^{\prime}+\frac{1}{6} \frac{B_{u}}{B}, \quad \mathfrak{S}^{\prime}=C^{\prime}+A^{\prime} B^{\prime}, \\
\mathfrak{I}^{\prime \prime} & =A^{\prime \prime}, & \mathfrak{S}^{\prime \prime} & =C^{\prime \prime}-A_{v}^{\prime}-2 A^{\prime 2}+A^{\prime \prime} B^{\prime} .
\end{aligned}
$$

In fact we have

$$
\begin{aligned}
& \overline{\mathfrak{B}}=\frac{V^{\prime}}{\left(U^{\prime}\right)^{2}} \mathfrak{B}, \quad \overline{\mathfrak{E}}=\frac{1}{\left(U^{\prime}\right)^{2}} \mathfrak{E}, \\
& \overline{\mathfrak{A}} \mathfrak{I}^{\prime}=\frac{1}{V^{\prime}}, \mathfrak{U}^{\prime}, \quad \overline{\mathfrak{B}^{\prime}}=\frac{1}{U^{\prime}}, \mathfrak{B}^{\prime}, \quad \overline{\mathfrak{S}^{\prime}}=\frac{1}{U^{\prime} V^{\prime}}, \mathfrak{S}^{\prime}, \\
& \overline{\mathfrak{A}}^{\prime \prime}=\frac{U^{\prime}}{\left(V^{\prime}\right)^{2}} \mathfrak{A}^{\prime \prime}, \quad \overline{\mathfrak{S}}^{\prime \prime}=\frac{1}{\left(V^{\prime}\right)^{2}} \mathfrak{E}^{\prime \prime} .
\end{aligned}
$$

Whenever a relative invariant $\theta^{(m, n)}$ satisfies the equation

$$
\bar{\theta}^{(m, n)}=\left(U^{\prime}\right)^{m}\left(V^{\prime}\right)^{n} \theta^{(m, n)}
$$

we shall say that its weights with respect to $u$ and $v$ are $m$ and $n$ respectively. These weights may be characterized by the symbol $(m, n)$. Thus $\mathfrak{B}$ is of weight $(-2,1)$, etc. We proceed to show how we may deduce from $\theta^{(m, n)}$ by differentiation two new invariants, which we shall denote by $\left(\theta^{(m, n)}, B^{\prime}\right)$ and $\left(\theta^{(m, n)}, A^{\prime}\right)$.

We find from (23)

$$
\begin{aligned}
& \bar{\theta}_{u}^{(m, n)}=\left(U^{\prime}\right)^{m-1}\left(V^{\prime}\right)^{n}\left(\theta_{u}^{(m, n)}+m \eta \theta^{(m, n)}\right), \\
& \bar{\theta}_{v}^{(m, n)}=\left(U^{\prime}\right)^{m}\left(V^{\prime}\right)^{n-1}\left(\theta_{v}^{(m, n)}+n \zeta \theta^{(m, n)}\right),
\end{aligned}
$$

where, of course, $\bar{\theta}_{u}^{(m, n)}$ and $\bar{\theta}_{v}^{(m, n)}$ denote $\partial \bar{\theta}^{(m, n)} / \partial \bar{u}$ and $\partial \bar{\theta}^{(m, n)} / \partial \bar{v}$ respectively. Since we have from (20)

$$
\bar{A}^{\prime}=\frac{1}{V^{\prime}}\left(A^{\prime}+\frac{1}{3} \zeta\right), \quad \bar{B}^{\prime}=\frac{1}{U^{\prime}}\left(B^{\prime}+\frac{1}{3} \eta\right),
$$


we shall find that the expressions

$$
\left(\theta^{(m, n)}, B^{\prime}\right)=\theta_{u}^{(m, n)}-3 m B^{\prime} \theta^{(m, n)}, \quad\left(\theta^{(m, n)}, A^{\prime}\right)=\theta_{v}^{(m, n)}-3 n A^{\prime} \theta^{(m, n)}
$$

are two new invariants of weights $(m-1, n)$ and $(m, n-1)$ respectively. It is also very easy to verify that if $\theta$ is an invariant (relative or absolute), $\partial^{2} \log \theta / \partial u \partial v$ is also an invariant, of weight $(-1,-1)$.

Clearly we may obtain from (22), by the repeated application of the two processes defined by equations (25), an infinite number of invariants. We wish to show that all invariants of the net will be functions of those obtained in this manner.

In the first place we recall the fact that every invariant is a function of seminvariants, i. e., of $B, C$, etc., and of the derivatives of these quantities. But (21) shows that $B, C, \ldots$ may be expressed as functions of $\mathfrak{B}, \mathfrak{C}, \mathfrak{A}^{\prime}, \mathfrak{B}^{\prime}$, $\mathfrak{C}^{\prime}, \mathfrak{\mathfrak { U } ^ { \prime \prime }}, \mathfrak{C}^{\prime \prime}$, and of their derivatives. Consequently every invariant $I$ must be a function of the seven fundamental invariants and of their derivatives, i. e.,

$$
I=F\left(\mathfrak{B}, \mathfrak{E}, \ldots ; \mathfrak{B}_{u}, \ldots ; \mathfrak{B}_{v}, \ldots ; \mathfrak{B}_{u u}, \cdots\right) .
$$

Let us introduce infinitesimal transformations by putting

$$
U=u+\phi(u) \delta t, \quad V=v+\psi(v) \delta t,
$$

where $\delta t$ is an infinitesimal. Then and

$$
U^{\prime}=1+\phi^{\prime} \delta t, \quad V^{\prime}=1+\psi^{\prime} \delta t, \quad \eta=\phi^{\prime \prime} \delta t, \quad \zeta=\psi^{\prime \prime} \delta t
$$

$$
\begin{aligned}
& \delta \mathfrak{B}=\left(-2 \phi^{\prime}+\psi^{\prime}\right) \mathfrak{B} \delta t, \quad \delta \mathfrak{E}=-2 \phi^{\prime} \mathfrak{E} \delta t, \\
& \delta \mathfrak{\mathfrak { U } ^ { \prime }}=-\boldsymbol{\psi}^{\prime} \mathfrak{A}^{\prime} \delta t, \quad \delta \mathfrak{B}^{\prime}=-\phi^{\prime} \mathfrak{B}^{\prime} \delta t, \quad \delta \mathfrak{S}^{\prime}=-\left(\phi^{\prime}+\psi^{\prime}\right) \mathfrak{C}^{\prime} \delta t, \\
& \delta \mathfrak{U}^{\prime \prime}=\left(\phi^{\prime}-2 \psi^{\prime}\right) \mathfrak{\mathfrak { U } ^ { \prime \prime }} \delta t, \quad \delta \mathfrak{C}^{\prime \prime}=-2 \psi^{\prime} \mathfrak{C}^{\prime \prime} \delta t .
\end{aligned}
$$

\begin{tabular}{|c|c|c|c|c|c|c|c|c|c|c|c|c|c|c|}
\hline & $\mathscr{P}_{u}$ & $\mathscr{B}_{v}$ & $\mathfrak{\Theta}_{u}$ & $\mathbb{E}_{v}$ & $\mathfrak{x}_{u}^{\prime}$ & $x_{v}^{\prime}$ & $\mathfrak{B}_{u}^{\prime}$ & $\mathbb{Z}_{v}^{\prime}$ & $\mathfrak{e}_{u}^{\prime}$ & $\mathfrak{e}_{v}^{\prime}$ & $\mathfrak{u}_{u}^{\prime \prime}$ & $\mathfrak{x}_{v}^{\prime \prime}$ & $\mathfrak{e}_{u}^{\prime \prime}$ & $\mathfrak{E}_{v}^{\prime \prime}$ \\
\hline$\phi^{\prime}$ & $-3 \mathfrak{P}_{u}$ & $-2 \mathfrak{B}_{v}$ & $\left|-3 \mathfrak{S}_{u}\right|$ & $-2 \mathbb{S}_{v}$ & $-\mathfrak{A}_{u}^{\prime}$ & & $-2 \mathfrak{B}_{u}^{\prime}$ & $-\mathfrak{B}_{v}^{\prime}$ & $-2 \mathfrak{S}_{u}^{\prime}$ & $-\mathfrak{E}_{v}^{\prime}$ & & $+\mathfrak{A}_{v}^{\prime \prime}$ & $-\mathfrak{E}_{u}^{\prime \prime}$ & \\
\hline$\psi^{\prime}$ & $+\mathfrak{B}_{u}$ & & & $-\mathfrak{E}_{v}$ & $-\mathfrak{A}_{u}^{\prime}$ & $-2 \mathfrak{X}_{v}^{\prime}$ & & $-\mathfrak{B}_{v}^{\prime}$ & $-\mathfrak{E}_{u}^{\prime}$ & $-2 \mathfrak{S}_{v}^{\prime}$ & $-2 \mathfrak{A}_{u}^{\prime \prime}$ & $-3 \mathfrak{A}_{v}^{\prime \prime}$ & $-2 \mathfrak{S}_{u}^{\prime \prime}$ & $-3 \mathfrak{E}_{v}^{\prime \prime}$ \\
\hline$\phi^{\prime \prime}$ & $-2 \mathfrak{B}$ & & $-2 \mathfrak{C}$ & & & & $-\mathfrak{B}^{\prime}$ & & $-\mathfrak{S}^{\prime}$ & & $+\mathfrak{A}^{\prime \prime}$ & & & \\
\hline$\psi^{\prime \prime}$ & & $+\mathscr{B}$ & & & & $-\mathfrak{A}^{\prime}$ & & & & $-\mathbb{E}^{\prime}$ & & $-2 \mathfrak{U}^{\prime \prime}$ & & $-2 \mathbb{S}^{\prime \prime}$ \\
\hline
\end{tabular}

In general, for an invariant $\theta^{(m, n)}$, of weight $(m, n)$, we shall find

$$
\begin{aligned}
& \delta \theta^{(m, n)}=\left(m \phi^{\prime}+n \psi^{\prime}\right) \theta^{(m, n)} \delta t, \\
& \delta \theta_{u}^{(m, n)}=\left[\left\{(m-1) \phi^{\prime}+n \psi^{\prime}\right\} \theta_{u}^{(m, n)}+m \theta^{(m, n)} \phi^{\prime \prime}\right] \delta t, \\
& \delta \theta_{v}^{(m, n)}=\left[\left\{m \phi^{\prime}+(n-1) \psi^{\prime}\right\} \theta_{v}^{(m, n)}+n \theta^{(m, n)} \psi^{\prime \prime}\right] \delta t .
\end{aligned}
$$

The latter formulæ enable us to calculate $\delta \mathfrak{F}_{u}, \delta \mathfrak{B}_{v}$, etc. The results may be tabulated as follows : 
Let $I$ be an invariant (absolute) which depends only upon the seven fundamental invariants and their first derivatives. Then $\delta I$ must vanish for arbitrary values of $\phi^{\prime}, \psi^{\prime}, \phi^{\prime \prime}, \psi^{\prime \prime}$. We thus obtain a system of four partial differential equations for $I$, involving 21 independent variables. The matrix of the coefficients of these four differential equations is obtained by adding the seven columns corresponding to the infinitesimal transformations of $\mathfrak{B}, \mathfrak{C}, \ldots, \mathfrak{S}^{\prime \prime}$ to the table just calculated. The form of the matrix makes it evident at once that the four equations are independent. Moreover, according to Lie's theory they must form a complete system. Therefore, there must exist $21-4=17$ independent absolute invariants of the kind in question. Five of these may be chosen as independent of the derivatives. In fact we have

so that

$$
\overline{\mathfrak{A}^{\prime \prime} \mathfrak{B}}=\frac{1}{\left(U^{\prime}\right)^{3}} \mathfrak{A}^{\prime \prime} \mathfrak{B}^{2}, \quad \overline{\mathfrak{U}^{\prime \prime 2} \mathfrak{B}}=\frac{1}{\left(V^{\prime}\right)^{3}} \mathfrak{I}^{\prime \prime 2} \mathfrak{B},
$$

$$
\frac{\mathfrak{C}^{3}}{\mathfrak{A}^{\prime \prime 2} \mathfrak{B}^{4}}, \frac{\mathfrak{A}^{\prime 3}}{\mathfrak{A}^{\prime \prime 2} \mathfrak{B}}, \frac{\mathfrak{B}^{\prime 3}}{\mathfrak{U}^{\prime \prime} \mathfrak{B}^{2}}, \frac{\mathfrak{C}^{\prime 3}}{\mathfrak{A}^{\prime \prime 3} \mathfrak{B}^{3}}, \frac{\mathfrak{C}^{\prime \prime 3}}{\mathfrak{A}^{\prime \prime 4} \mathfrak{B}^{2}}
$$

are five independent absolute invariants. From the fundamental seven relative invariants we can form fourteen others of the form $\left(\theta^{(m . n)}, B^{\prime}\right)$ and $\left(\theta^{(m, n)}, A^{\prime}\right)$, but, of course, only twelve of these can be independent. In fact the following two,

$$
\left(\mathfrak{B}, B^{\prime}\right), \quad\left(\mathfrak{U}^{\prime \prime}, A^{\prime}\right)
$$

reduce to $6 \mathfrak{B} \mathfrak{B}^{\prime}$ and $6 \mathfrak{U}^{\prime} \mathfrak{U}^{\prime \prime}$ respectively, while the other twelve are independent of each other and of the fundamental seven.

We have proved that all invariants involving only the first derivatives of $\mathfrak{B}$, $\mathbb{E}$, etc., may be obtained from the fundamental seven by the processes (25). Let us speak of these invariants as being of the first order. Now let $I$ be an invariant of the second order. The system of differential equations satisfied by such invariants contains two more equations than the corresponding system for invariants of the first order. The matrix of the coefficients of $\partial I / \partial \mathfrak{B}, \partial I / \partial \mathscr{U} \mathfrak{U}^{\prime \prime}$, $\partial I / \partial \mathfrak{B}_{v}, \partial I / \partial \mathfrak{U}_{u}^{\prime \prime}, \partial I / \partial \mathfrak{B}_{v v}, \partial I / \partial \mathfrak{U}_{v v}^{\prime \prime}$ may be represented by means of the matrices

in the form

$$
M=\left\|\begin{array}{ll}
-2 \mathfrak{B}, & +\mathfrak{U}^{\prime \prime} \\
+\mathfrak{B}, & -2 \mathfrak{U}^{\prime \prime}
\end{array} \mid, \quad N=\right\| \begin{array}{rr}
0, & +\mathfrak{U}^{\prime \prime} \\
+\mathfrak{B}, & 0
\end{array} \|
$$

$$
\left|\begin{array}{ccc}
M, & P, & Q \\
0, & N, & R \\
0, & 0, & N
\end{array}\right|
$$

where 0 denotes a square matrix of the second order with vanishing elements, and where the values of the coefficients of the second order matrices $P, Q, R$ 
are immaterial. The determinant of this matrix is

where

$$
|M||N|^{2}
$$

$$
|M|=3 \mathfrak{U}{ }^{\prime \prime} \mathfrak{B}, \quad|N|=-\mathfrak{U}^{\prime \prime} \mathfrak{B},
$$

and therefore, in general, different from zero. The two new equations of the system are therefore independent. The same proof applies to the two new equations obtained when we pass to the invariants of the third order, etc.

The system of differential equations for the invariants of the second order contains 21 more independent variables and 2 more independent equations than that for invariants of the first order. There must exist, therefore, 19 independent invariants of the second order. The application of our two processes to the twelve independent invariants of the first order gives 24 invariants of the second order. We proceed to show that just nineteen of these are independent, so that our process actually furnishes them all.

We have

where

$$
\left(\mathfrak{B}, A^{\prime}\right)=\mathfrak{V}_{v}-3 A^{\prime} \mathfrak{B}, \quad\left(\mathfrak{U}^{\prime \prime}, B^{\prime}\right)=\mathfrak{U}_{u}^{\prime \prime}-3 B^{\prime} \mathfrak{U}^{\prime \prime},
$$

$$
\begin{aligned}
{\left[\left(\mathfrak{E}, A^{\prime}\right), B^{\prime}\right) } & =\mathfrak{V}_{u v}-3 A^{\prime} \mathfrak{B}_{u}-3 A_{u}^{\prime} \mathfrak{B}+6 B^{\prime}\left(\mathfrak{B}, A^{\prime}\right), \\
{\left[\left(\mathfrak{U}^{\prime \prime}, B^{\prime}\right), A^{\prime}\right) } & =\mathfrak{U}_{u v}^{\prime \prime}-3 B^{\prime} \mathfrak{U}_{v}^{\prime \prime}-3 B_{v}^{\prime} \mathfrak{A}^{\prime \prime}+6 A^{\prime}\left(\mathfrak{U}^{\prime \prime}, B^{\prime}\right) .
\end{aligned}
$$

If we remember that

$$
A^{\prime}=\mathfrak{A} \mathfrak{A}^{\prime}-\frac{1}{6} \frac{\mathfrak{\mathfrak { A } _ { v } ^ { \prime \prime }}}{\mathfrak{U}^{\prime \prime}}, \quad B^{\prime}=\mathfrak{B}^{\prime}-\frac{1}{6} \frac{\mathfrak{B}_{u}}{\mathfrak{B}},
$$

we see that these invariants contain the second derivatives of the fundamental invariants only in the combinations

$$
\mathfrak{B}_{u v}+\frac{1}{2} \frac{\mathfrak{B}}{\mathfrak{A}^{\prime \prime}} \mathfrak{A}_{u v}^{\prime \prime} \quad \text { and } \quad \mathfrak{A}_{u v}^{\prime \prime}+\frac{1}{2} \frac{\mathfrak{Q}^{\prime \prime}}{\mathfrak{B}} \mathfrak{B}_{u v}
$$

respectively, and are, therefore, independent. The other two invariants of the second order which can be formed from $\mathfrak{U}^{\prime \prime}$ and $\mathfrak{B}$, viz.,

$$
\left[\left(\mathfrak{B}, A^{\prime}\right), A^{\prime}\right] \quad \text { and } \quad\left[\left(\mathfrak{A}^{\prime \prime}, B^{\prime}\right), B^{\prime}\right]
$$

contain the further variables $\mathfrak{B}_{v v} \mathfrak{A}_{v v}^{\prime \prime}$ and $\mathfrak{B}_{u u} \mathfrak{A}_{u u}^{\prime \prime}$ respectively, so that these four are certainly independent.

Each of the remaining five pairs of invariants of the first order gives rise to only three instead of four independent invariants of the second order. We have, for example,

$$
\left(\mathfrak{C}, B^{\prime}\right)=\mathfrak{C}_{u}+6 B^{\prime} \mathfrak{C}, \quad\left(\mathfrak{C}, A^{\prime}\right)=\mathfrak{C}_{v}
$$

so that $\left[\left(\mathfrak{S}, B^{\prime}\right), B^{\prime}\right]$ and $\left[\left(\mathfrak{S}, A^{\prime}\right), A^{\prime}\right]$ are obviously independent. But

$$
\begin{aligned}
& {\left[\left(\mathfrak{S}, B^{\prime}\right), A^{\prime}\right]=\mathfrak{C}_{u v}+6 B^{\prime} \mathfrak{S}_{v}+6 B_{v}^{\prime} \mathfrak{E},} \\
& {\left[\left(\mathfrak{E}, A^{\prime}\right), B^{\prime}\right]=\mathfrak{E}_{u v}+6 B^{\prime} \mathfrak{E}_{v},}
\end{aligned}
$$


so that their difference involves, of second order derivatives, only $\mathfrak{B}_{u v}$ which by our previous consideration, is capable of expression in terms of $\left[(\mathfrak{B}, A), B^{\prime}\right]$, $\left[\left(\mathfrak{U}^{\prime \prime}, B^{\prime}\right), A^{\prime}\right]$ and invariants of lower order. Our process actually supplies us, therefore, with $2.2+5.3=19$ independent invariants of the second order.

It is clear that there must exist $7 n+5$ independent invariants of the $n$th order (not counting those of order lower than $n$ ). Let us assume that $n$ of these, those obtained from $\mathfrak{B}$ by our differentiation processes, are of the form

$$
\mathfrak{B}_{n-k, k}=\frac{\partial^{n} \mathfrak{B}}{\partial u^{n-k} \partial v^{k}}+\frac{\mathfrak{B}}{2 \mathfrak{U}^{\prime \prime}} \frac{\partial^{n} \mathfrak{U}^{\prime \prime}}{\partial u^{n-k}} \overline{\partial v^{k}}+\cdots \quad(k=1,2, \cdots, n),
$$

the terms not written being of order lower than $n$. Then none of these $n$ invariants involves $\partial^{n} \mathfrak{B} / \partial u^{n}$ or $\partial^{n} \mathfrak{Q}^{\prime \prime} / \partial u^{n}$. Let $n$ other invariants be of the form

$$
\mathfrak{A}_{n-k, k}^{\prime \prime}=\frac{\partial^{n} \mathfrak{U}^{\prime \prime}}{\partial u^{n-k} \partial v^{k}}+\frac{\mathfrak{U}^{\prime \prime}}{2 \mathfrak{V}} \frac{\partial^{n} \mathfrak{B}}{\partial u^{n-k} \partial v^{k}}+\cdots \quad(k=0,1,2, \cdots, n-1),
$$

so that none of these involves the variables $\partial^{n} \mathfrak{U}^{\prime \prime} / \partial v^{n}$ or $\partial^{n} \mathfrak{B} / \partial v^{n}$. Let the invariants formed from $\mathfrak{E}, n+1$ in number, be

$$
\mathfrak{S}_{n-k, k}=\frac{\partial^{n} \mathfrak{E}}{\partial u^{n-k} \partial v^{k}}+\cdots \quad(k=0,1,2, \cdots, n),
$$

where the omitted terms may involve, of quantities of the $n$th order, only the derivatives of $\mathfrak{U}^{\prime \prime}$ and $\mathfrak{B}$. Let there be $n+1$ invariants of this same character for each of the remaining fundamental invariants $\mathfrak{A}^{\prime}, \mathfrak{B}^{\prime}, \mathfrak{C}^{\prime}$ and $\mathfrak{C}^{\prime \prime}$. All of these conditions are actually satisfied for $n=2$.

Let us consider the invariants of order $n+1$,

$$
\begin{aligned}
& \left(\mathfrak{B}_{n-k, k}, B^{\prime}\right), \quad k=1,2, \ldots, n, \quad\left(\mathfrak{B}_{0, n}, A^{\prime}\right), \\
& \left(\mathfrak{U}_{n-k, k}^{\prime \prime}, A^{\prime}\right), \quad k=0,1,2, \ldots, n-1, \quad\left(\mathfrak{A}_{n, 0}^{\prime \prime}, B^{\prime}\right), \\
& \left(\mathfrak{C}_{n-k, k}, B^{\prime}\right), \quad k=0,1,2, \cdots, n, \quad\left(\mathfrak{E}_{0, n}, A^{\prime}\right),
\end{aligned}
$$

and the corresponding ones formed from $\mathfrak{A}^{\prime}, \mathfrak{B}^{\prime}, \mathfrak{E}^{\prime}, \mathfrak{E}^{\prime \prime}$. They will be $2(n+1)+5(n+2)=7 n+12$ in number; they will obviously be independent and will have all of the properties which were assumed for the invariants of order $n$. Since there exists a system of invariants of the second order with these properties, there exists a system of invariants of the $n$th order with the same properties, as our induction proof clearly shows. Since, moreover, our process gives us precisely the right number of independent invariants of order $n+1$, we have proved the following theorem.

Every invariant of the system of partial differential equations (4) is a function of the fundamental seven, and of those others which can be obtained from them by repeated application of the two differentiation processes (25).

The system of equations (14), the integrability conditions for (4), must of 
course be an invariant system of equations. We find, more specifically, that each one of them is invariant by itself. It must therefore be possible to rewrite these conditions in such a form as to exhibit their invariant character. In order to do this in a convenient form we shall introduce the two further invariants

$$
H=C^{\prime}+A^{\prime} B^{\prime}-A_{u}^{\prime}, \quad K=C^{\prime}+A^{\prime} B^{\prime}-B_{v}^{\prime},
$$

which, of course, may be expressed in terms of our fundamental invariants, but which merit a special notation on account of their great importance, and because their expression in terms of the fundamental invariants is rather complicated. We shall first show how these latter expressions may be obtained. Since

we have

$$
\begin{aligned}
A_{u}^{\prime} & =\mathfrak{A}_{u}^{\prime}-\frac{1}{6} \frac{\partial^{2} \log \mathfrak{A}^{\prime \prime}}{\partial u \partial v}, & B_{v}^{\prime} & =\mathfrak{B}_{v}^{\prime}-\frac{1}{6} \frac{\partial^{2} \log \mathfrak{B}}{\partial u \partial v} \\
\mathfrak{A}_{u}^{\prime} & =\left(\mathfrak{A}^{\prime}, B^{\prime}\right), & \mathfrak{B}_{v}^{\prime} & =\left(\mathfrak{B}^{\prime}, A^{\prime}\right),
\end{aligned}
$$

(31) $H=\mathfrak{C}^{\prime}-\left(\mathfrak{A}^{\prime}, B^{\prime}\right)+\frac{1}{6} \frac{\partial^{2} \log \mathfrak{A}^{\prime \prime}}{\partial u \partial v}, \quad K=\mathfrak{C}^{\prime}-\left(\mathfrak{B}^{\prime}, A^{\prime}\right)+\frac{1}{6} \frac{\partial^{2} \log \mathfrak{B}}{\partial u \partial v}$,

so that it remains to find the expressions for $\partial^{2} \log \mathfrak{A}^{\prime \prime} / \partial u \partial v$ and $\partial^{2} \log \mathfrak{B} / \partial u \partial v$. Now we have

$\left(\mathfrak{B}, A^{\prime}\right)=\mathfrak{B}_{v}-3 A^{\prime} \mathfrak{B}, \quad\left[\left(\mathfrak{B}, A^{\prime}\right), B^{\prime}\right]=\mathfrak{B}_{u v}-3 A^{\prime} \mathfrak{B}_{u}-3 A_{u}^{\prime} \mathfrak{B}+6 B^{\prime}\left(\mathfrak{B}, A^{\prime}\right)$.

From these equations we find

$$
2 \frac{\partial^{2} \log \mathfrak{B}}{\partial u \partial v}+\frac{\partial^{2} \log \mathfrak{A}^{\prime \prime}}{\partial u \partial v}=\frac{1}{\mathfrak{B}}\left\{2\left[\left(\mathfrak{B}, A^{\prime}\right), B^{\prime}\right]-12 \mathfrak{B}^{\prime}\left(\mathfrak{B}, A^{\prime}\right)+6 \mathfrak{B}\left(\mathfrak{U}^{\prime}, B^{\prime}\right)\right\}
$$

In the same way we may prove the formula

$$
\frac{\partial^{2} \log \mathfrak{B}}{\partial u \partial v}+2 \frac{\partial^{2} \log \mathfrak{A}^{\prime \prime}}{\partial u \partial v}=\frac{1}{\mathfrak{U}^{\prime \prime}}\left\{2\left[\left(\mathfrak{U}^{\prime \prime}, B^{\prime}\right), A^{\prime}\right]-12 \mathfrak{U}^{\prime}\left(\mathfrak{A}^{\prime \prime}, B^{\prime}\right)+6 \mathfrak{U}^{\prime \prime}\left(\mathfrak{B}^{\prime}, A^{\prime}\right)\right\}
$$

and these two equations suffice to solve our problem.

The integrability conditions may now be written as follows :

$$
\begin{aligned}
3-\mathfrak{U}^{\prime \prime} \mathfrak{B}-H-K & =0, \\
\mathfrak{C}+\left(\mathfrak{B}, A^{\prime}\right) & =0, \\
\mathfrak{S}^{\prime \prime}+\left(\mathfrak{U}^{\prime \prime}, B^{\prime}\right) & =0, \quad\left(\mathfrak{C}, A^{\prime}\right)-\left(K, B^{\prime}\right)+\mathfrak{B} \mathfrak{C}^{\prime \prime}=0, \\
\left(\mathfrak{S}^{\prime \prime}, B^{\prime}\right)-\left(H, A^{\prime}\right)+\mathfrak{U}^{\prime \prime} \mathfrak{C}=0, &
\end{aligned}
$$

and in this form their invariant character is obvious.

We have already observed that the seminvariants are capable of being expressed in terms of the fundamental invariants and of their derivatives. But if the seminvariants are known as functions of $u$ and $v$ the corresponding net of plane curves is determined except for a projective transformation. We see, therefore, that the following theorem is true.

If the seven fundamental invariants of a net are given as functions of $u$ and 
v so as to satisfy the five conditions (34), the net is determined except for a projective transformation.

\section{§4. The covariants of the net and its Laplacian Transformations.}

Let

$$
y^{(k)}=f^{(k)}(u, v) \quad(k=1,2,3)
$$

be the equations of the net of plane curves under consideration. For given values of $u$ and $v, y^{(1)}, y^{(2)}, y^{(3)}$ are the coördinates of a point $y^{*}$ through which pass the two curves $u=$ const. and $v=$ const. of our net. The tangent $t$ to the curve $v=$ const. will be obtained by joining $y$ to the point $y_{u}$ whose coördinates are

$$
y_{u}^{(k)}=\frac{\partial f^{(k)}(u, v)}{\partial u} \quad(k=1,2,3),
$$

and the coördinates of any point of this tangent (distinct from $y$ ) may be expressed in the form

$$
y_{u}^{(k)}+\alpha y^{(k)} \quad(k=1,2,3) .
$$

Now let $y$ move along a curve $u=$ const., and in each of its positions construct the line $t$ tangent to that one of the curves $v=$ const. which passes through it. These lines $t$ will have an envelope, which we now proceed to determine. The expressions (35) are the coördinates of an arbitrary point $Q$ of $t$. As $y$ moves along the curve $u=$ const., $v$ alone changes, so that the tangent of the curve described by $Q$ will be obtained by joining $Q$ to the point $R$ whose coördinates are

$$
\begin{aligned}
\frac{\partial}{\partial v}\left(y_{u}^{(k)}+\alpha y^{(k)}\right) & =y_{u v}^{(k)}+\alpha y_{v}^{(k)}+\alpha_{v} y^{(k)} \\
& =a^{\prime} y_{u}^{(k)}+\left(b^{\prime}+\alpha\right) y_{v}^{(k)}+\left(c^{\prime}+\alpha_{v}\right) y^{(k)} \quad(k=1,2,3) .
\end{aligned}
$$

Now if $Q$ is the point where $t$ meets its envelope, $t$ must be tangent to the envelope at $Q$, i. e., the line $Q R$ must coincide with $t$. This will be so if and only if $\alpha=-b^{\prime}$, assuming of course that the curves $u=$ const. and $v=$ const. are not tangent to each other at the point $y$.

We see, therefore, that the tangents to the curves $v=$ const. constructed at the points where these curves meet a fixed curve $u=c o n s t$. have as their envelope the locus of the point $P_{\rho}$ whose coördinates are

$$
\rho^{(k)}=y_{u}^{(k)}-b^{\prime} y^{(k)} \quad(k=1,2,3) .
$$

Similarly, the tangents to the curves $u=$ const. along a ficed curve $v=$ const., have as their envelope the locus of the point $P_{\sigma}$ whose coördinates are given by

$$
\sigma^{(k)}=y_{v}^{(k)}-a^{\prime} y^{(k)} \quad(k=1,2,3) .
$$

* This is, of course, the same point which has previously been denoted by $P_{y}$. 
It follows from these considerations, and may easily be verified analytically that the semi-covariants

$$
\rho=y_{u}-b^{\prime} y, \quad \sigma=y_{\mathrm{v}}-a^{\prime} y
$$

are also covariants of system (4). The same thing is, of course, true of $y$ itself.

All other relative covariants are homogeneous functions of these three and of invariants. For in the first place we need consider only covariants which involve, necessarily in homogeneous fashion, $y, y_{4}$ and $y_{0}$ and no derivatives of $y$ of a higher order. In fact, if a covariant did involve higher derivatives we could express them in terms of these three by means of equations (4) and those derived from them by differentiation. If we were to set up the system of partial differential equations satisfied by the absolute covariants, we should find the same system as that considered before for the absolute invariants with the additional terms arising from the presence of the two new variables, viz., the two ratios of the quantities $y, y_{u}$ and $y_{v}$. This system will therefore have two more independent solutions than that for the invariants, and these two may be chosen to be $\rho / y$ and $\sigma / y$ since they are clearly independent. All absolute covariants, then, are functions of $\rho / y, \sigma / y$ and of absolute invariants, whence follows at once the theorem as stated.

The covariants $\rho$ and $\sigma$, as we have seen, establish a correspondence between the point $P_{y}$ and two other points $P_{\rho}$ and $P_{\sigma}$. As $u$ and $v$ vary, each of these two points $P_{\rho}$ and $P_{\sigma}$, in general, describes a new net of plane curves which shall be called Laplacian transforms of the net determined by $P_{y}$. More specifically we shall call the net determined by $P_{\sigma}$ the first, and that determined by $P_{\rho}$ the minus first Laplacian transform of the original net, for the reason that the first Laplacian transform of the net determined by $P_{\rho}$ is the original net. We proceed to set up the partial differential equations of the net determined by $P_{\sigma}$.

We may assume that the system (4) has been reduced to its canonical form

$$
\begin{aligned}
& y_{u u}=-B^{\prime} y_{u}+B y_{v}+C y, \\
& y_{u v}=A^{\prime} y_{u}+B^{\prime} y_{v}+C^{\prime} y, \\
& y_{v v}=A^{\prime \prime} y_{u}-A^{\prime} y_{v}+C^{\prime \prime} y .
\end{aligned}
$$

We shall find from (36), by differentiation and making use of (37),

$$
\sigma_{u}=B^{\prime} y_{v}+\left(C^{\prime}-A_{u}^{\prime}\right) y, \quad \sigma_{v}=A^{\prime \prime} y_{u}-2 A^{\prime} y_{v}+\left(C^{\prime \prime}-A_{v}^{\prime}\right) y,
$$

and further

$$
\begin{aligned}
& \sigma_{u u}=\left(C^{\prime}-A_{u}^{\prime}+A^{\prime} B^{\prime}\right) y_{u}+\left(B_{u}^{\prime}+B^{\prime 2}\right) y_{v}+\left(C_{u}^{\prime}-A_{u u}^{\prime}+B^{\prime} C^{\prime}\right) y, \\
& \sigma_{u v}= A^{\prime \prime} B^{\prime} y_{u}+\left(C^{\prime}-A_{u}^{\prime}+B_{v}^{\prime}-A^{\prime} B^{\prime}\right) y_{v}+\left(C_{v}^{\prime}-A_{u v}^{\prime}+B^{\prime} C^{\prime \prime}\right) y, \\
& \sigma_{v v}=\left(A_{v}^{\prime \prime}-A^{\prime} A^{\prime \prime}\right) y_{u}+\left(C^{\prime \prime}-3 A_{v}^{\prime}+A^{\prime \prime} B^{\prime}-2 A^{\prime} B^{\prime \prime}\right) y_{v} \\
& \quad+\left(C_{v}^{\prime \prime}-A_{v v}^{\prime}+A^{\prime \prime} C^{\prime}-2 A^{\prime} C^{\prime \prime}\right) y .
\end{aligned}
$$

Trans. Am. Math. Soc. 33 
Now from (36) and (38) we find

$$
\begin{aligned}
& y_{\diamond}=A^{\prime} y+\sigma, \\
& \sigma_{u}=H y+B^{\prime} \sigma, \quad \sigma_{v}=\left(C^{\prime \prime}-A_{v}^{\prime}-2 A^{\prime 2}\right) y+A^{\prime \prime} y_{u}-2 A^{\prime} \sigma,
\end{aligned}
$$

whence

$$
\text { (41) } \begin{aligned}
A^{\prime \prime} H y_{u} & =-\left(C^{\prime \prime}-A_{v}^{\prime}-2 A^{\prime 2}\right) \sigma_{u}+H \sigma_{v}+\left[2 H A^{\prime}+B^{\prime}\left(C^{\prime \prime}-A_{v}^{\prime}-2 A^{\prime 2}\right)\right] \sigma, \\
A^{\prime \prime} H y_{v} & =A^{\prime} A^{\prime \prime} \sigma_{u}+A^{\prime \prime}\left(H-A^{\prime} B^{\prime}\right) \sigma .
\end{aligned}
$$

We shall therefore obtain for $\sigma$ a system of partial differential equations of the form

$$
\begin{aligned}
& \sigma_{u u}=a_{1} \sigma_{u}+b_{1} \sigma_{v}+c_{1} \sigma, \\
& \sigma_{u v}=a_{1}^{\prime} \sigma_{u}+b_{1}^{\prime} \sigma_{v}+c_{1}^{\prime} \sigma, \\
& \sigma_{v v}=a_{1}^{\prime \prime} \sigma_{u}+b_{1}^{\prime \prime} \sigma_{v}+c_{1}^{\prime \prime} \sigma,
\end{aligned}
$$

whose coefficients may be expressed as follows :

$$
\begin{aligned}
& A^{\prime \prime} H a_{1}=A_{u}^{\prime \prime} H+A^{\prime \prime} H_{u}-A^{\prime \prime} H B^{\prime}, \quad A^{\prime \prime} H b_{1}=H^{2}, \\
& A^{\prime \prime} H c_{1}=-A^{\prime \prime} B^{\prime} H_{u}+2 A^{\prime} H^{2}+H\left(A^{\prime \prime} B_{u}^{\prime}-B^{\prime} A_{u}^{\prime \prime}+2 A^{\prime \prime} B^{2}\right), \\
& A^{\prime \prime} H a_{1}^{\prime}=A^{\prime \prime}\left(A^{\prime} H+H_{v}\right), \quad A^{\prime \prime} H b_{1}^{\prime}=A^{\prime \prime} B^{\prime} H, \\
& A^{\prime \prime} H c_{1}^{\prime}=-A^{\prime \prime} B^{\prime} H_{v}+A^{\prime \prime} H^{2}+A^{\prime \prime} H\left(B_{v}^{\prime}-A^{\prime} B^{\prime}\right), \\
& A^{\prime \prime} H a_{1}^{\prime \prime}=A^{\prime \prime} K+A^{\prime \prime} \mathfrak{S}_{v}^{\prime \prime}-\mathfrak{S}^{\prime \prime} A_{v}^{\prime \prime}, \quad A^{\prime \prime} H b_{1}^{\prime \prime}=\left(A_{v}^{\prime \prime}-A^{\prime} A^{\prime \prime}\right) H, \\
& A^{\prime \prime} H c_{1}^{\prime \prime}=A^{\prime \prime} H \mathfrak{S}-B^{\prime}\left(A^{\prime \prime} \mathfrak{S}_{v}^{\prime \prime}-\mathfrak{S}^{\prime \prime} A_{v}^{\prime \prime}+A^{\prime \prime 2} K\right)+2 H\left(A^{\prime} A_{v}^{\prime \prime}-A^{\prime \prime} A_{v}^{\prime}+A^{\prime 2} A^{\prime \prime}\right) .
\end{aligned}
$$

The system (42) is not in its canonical form. In order to reduce it to the canonical form, we put

$$
\sigma=\lambda y_{1}
$$

where $\lambda$ is subject to the conditions

$$
\frac{\lambda_{u}}{\lambda}=\frac{1}{3}\left(a_{1}+b_{1}^{\prime}\right)=\frac{1}{3}\left(\frac{A_{u}^{\prime \prime}}{A^{\prime \prime}}+\frac{H_{u}}{H}\right), \quad \frac{\lambda_{v}}{\lambda}=\frac{1}{3}\left(a_{1}^{\prime}+b_{1}^{\prime \prime}\right)=\frac{1}{3}\left(\frac{A_{v}^{\prime \prime}}{A^{\prime \prime}}+\frac{H_{v}}{H}\right),
$$

so that

$$
\lambda=\text { const. } \sqrt[3]{A^{\prime \prime} H}
$$

We shall omit the details of the calculation. A system of form (37) is transformed into another system likewise in its canonical form by the transformation

$$
y_{1}=\frac{y_{v}-A^{\prime} y}{\sqrt{A^{\prime \prime} H}} .
$$

Its coefficients, the seminvariants of the first Laplacian transformed net, are as 
follows :

$$
\begin{aligned}
& A_{1}=-B^{\prime}+\frac{1}{3}\left(\frac{A_{u}^{\prime \prime}}{A^{\prime \prime}}+\frac{H_{u}}{H}\right), \quad B_{1}=\frac{H}{A^{\prime \prime}}, \\
& C_{1}=B_{u}^{\prime}+2 B^{\prime 2}+2 \frac{A^{\prime} H}{A^{\prime \prime}}-\frac{4}{3} B^{\prime}\left(\frac{A_{u}^{\prime \prime}}{A^{\prime \prime}}+\frac{H_{u}}{H}\right)+\frac{1}{3} \frac{H}{A^{\prime \prime}}\left(\frac{A_{v}^{\prime \prime}}{A^{\prime \prime}}+\frac{H_{v}}{H}\right) \\
& +\frac{5}{9}\left(\frac{A_{u}^{\prime \prime}}{A^{\prime \prime}}+\frac{H_{u}}{\bar{H}}\right)^{2}-\frac{2}{3} \frac{A_{u}^{\prime \prime} H_{u}}{A^{\prime \prime} H}-\frac{1}{3}\left(\frac{A_{u u}^{\prime \prime}}{A^{\prime \prime}}+\frac{H_{u u}}{H^{\prime}}\right), \\
& A_{1}^{\prime}=A^{\prime}+\frac{H_{v}}{H}-\frac{1}{3}\left(\frac{A_{v}^{\prime \prime}}{\bar{A}^{\prime \prime}}+\frac{H_{v}}{H}\right), \quad B_{1}^{\prime}=B^{\prime}-\frac{1}{3}\left(\frac{A_{u}^{\prime \prime}}{A^{\prime \prime}}+\frac{H_{u}}{H}\right), \\
& C_{1}^{\prime}=C^{\prime}+B_{v}^{\prime}-A_{u}^{\prime}-B^{\prime} \frac{H_{v}}{H}+\frac{1}{3}\left(A^{\prime}+\frac{H_{v}}{H}\right)\left(\frac{A_{u}^{\prime \prime}}{A^{\prime \prime}}+\frac{H_{u}}{H}\right)+\frac{1}{3} B^{\prime}\left(\frac{A_{v}^{\prime \prime}}{A^{\prime \prime}}+\frac{H_{v}}{H}\right) \\
& -\frac{1}{9}\left(\frac{A_{u}^{\prime \prime}}{A^{\prime \prime}}+\frac{H_{u}}{H}\right)\left(\frac{A_{v}^{\prime \prime}}{A^{\prime \prime}}+\frac{H_{v}}{H}\right)-\frac{1}{3}\left(\frac{A_{u v}^{\prime \prime}}{A^{\prime \prime}}+\frac{H_{u v}}{H^{-}}-\frac{A_{u}^{\prime \prime} A_{v}^{\prime \prime}}{A^{\prime \prime 2}}-\frac{H_{u} H_{v}}{H^{2}}\right), \\
& A_{1}^{\prime \prime}=\frac{A^{\prime \prime} K}{H}+\frac{1}{H}\left(\mathfrak{C}_{v}^{\prime \prime}-\mathfrak{C}^{\prime \prime} \frac{A_{v}^{\prime \prime}}{A^{\prime \prime}}\right), \quad B_{1}^{\prime \prime}=-A^{\prime}+\frac{A_{v}^{\prime \prime}}{A^{\prime \prime}}-\frac{2}{3}\left(\frac{A_{v}^{\prime \prime}}{A^{\prime \prime}}+\frac{H_{v}}{H}\right), \\
& C_{1}^{\prime \prime}=\mathfrak{C}^{\prime \prime}-\frac{B^{\prime}}{H}\left(\mathfrak{C}_{v}^{\prime \prime}-\mathfrak{C}^{\prime \prime} \frac{A_{v}^{\prime \prime}}{A^{\prime \prime}}+K A^{\prime \prime}\right)+2\left(A^{\prime} \frac{A_{v}^{\prime \prime}}{A^{\prime \prime}}-A_{v}^{\prime}+A^{\prime 2}\right) \\
& +\frac{1}{3 H}\left(\mathfrak{S}_{v}^{\prime \prime}-\mathbb{C}^{\prime \prime} \frac{A_{v}^{\prime \prime}}{A^{\prime \prime}}+K A^{\prime \prime}\right)\left(\frac{A_{u}^{\prime \prime}}{A^{\prime \prime}}+\frac{H_{u}}{H}\right)+\frac{1}{3}\left(-A^{\prime}+\frac{A_{v}^{\prime \prime}}{A^{\prime \prime}}\right)\left(\frac{A_{v}^{\prime \prime}}{A^{\prime \prime}}+\frac{H_{v}}{H}\right) \\
& -\frac{1}{9}\left(\frac{A_{v}^{\prime \prime}}{A^{\prime \prime}}+\frac{H_{v}}{H}\right)^{2}-\frac{1}{3}\left[\frac{A_{v v}^{\prime \prime}}{A^{\prime \prime}}+\frac{H_{v v}}{H}-\left(\frac{A_{v}^{\prime \prime}}{A^{\prime \prime}}\right)^{2}-\left(\frac{H_{v}}{H}\right)^{2}\right] \text {, }
\end{aligned}
$$

where we have assumed $A^{\prime \prime}$ and $H$ different from zero. The cases thus excluded are necessarily so, since the corresponding net is degenerate. In fact, if $A^{\prime \prime}=0$, we see from (37) that

$$
y_{v v}=-A^{\prime} y_{v}+C^{\prime \prime} y
$$

so that the three solutions $y^{(k)}$ will satisfy a homogeneous relation of the first degree with coefficients independent of $v$; i. e., the curves $u=$ const. of the original net are straight lines. The locus of $P_{\sigma}$ in that case obviously degenerates into a curve, the envelope of these straight lines. This is also apparent from (40), for if $A^{\prime \prime}=0$, on account of $(14), C^{\prime \prime}-A_{v}^{\prime}-2 A^{\prime 2}$ will also vanish, so that we shall find

$$
\sigma_{v}=-2 A^{\prime} \sigma \text {. }
$$

The curves $u=$ const. of the transformed net degenerate into the points of the envelope, the curves $v=$ const. of the transformed net all coincide with this same envelope.

If $H=0$, equations (40) show that

$$
\sigma_{u}=B^{\prime} \sigma
$$


i. e., the envelope of the tangents to the curves $u=$ const. along a curve $v=$ const. degenerates into a point. In this case the curves $v=$ const. of the transformed net degenerate into points, and the curves $u=$ const. all coincide with the locus of these points.

We can now verify the following theorem. If $A^{\prime \prime}=0$ or $B=0$, one of the families of the original net is composed of straight lines and the first or minus first Laplacian transformed net degenerates. The only other cases in which one of these transformed nets degenerates are obtained by equating either $H$ or $K$ to zero.

In fact, the first Laplacian transformed net will degenerate if and only if the locus of $P_{\sigma}$ for all possible values of $u$ and $v$ reduces to a curve, i. e., if $\sigma$ satisfies an equation of the form

or if

$$
\alpha \sigma_{u}+\beta \sigma_{v}+\gamma \sigma=0
$$

$$
\alpha\left(H y+B^{\prime} \sigma\right)+\beta\left[\left(C^{\prime \prime}-A_{v}^{\prime}-2 A^{\prime 2}\right) y+A^{\prime \prime} y_{u}-2 A^{\prime} \sigma\right]+\gamma \sigma=0 .
$$

Now the points $y, y_{u}$ and $\sigma$ are not collinear if the curves $u=$ const. and $v=$ const. are not tangent to each other at the point $P_{y}$. Consequently we should have

$$
\alpha H+\beta\left(C^{\prime \prime}-A_{v}^{\prime}-2 A^{\prime 2}\right)=0, \quad \beta A^{\prime \prime}=0, \quad \alpha B^{\prime}-2 \beta A^{\prime}+\gamma=0 .
$$

If $H$ is not zero, we should therefore find necessarily $A^{\prime \prime}=0$, since $\alpha=\beta=\gamma=0$ is not an admissible solution.

The seven fundamental invariants of the first Laplacian transformed net have the following values :

$$
\begin{aligned}
& \mathfrak{B}_{1}=\frac{H}{\mathfrak{A}^{\prime \prime}}, \quad \mathfrak{C}_{1}=\frac{1}{\mathfrak{A}^{\prime \prime}}\left(H_{v}+3 A^{\prime} H\right), \\
& \mathfrak{A}_{1}^{\prime}=A^{\prime}+\frac{2}{3} \frac{H_{v}}{H}-\frac{1}{3} \frac{\mathfrak{U}_{v}^{\prime \prime}}{\mathfrak{U}^{\prime \prime}}+\frac{1}{6 \mathfrak{\mathfrak { U } _ { 1 } ^ { \prime \prime }}} \frac{\partial \mathfrak{Q}_{1}^{\prime \prime}}{\partial v}, \quad \mathfrak{B}_{1}^{\prime}=B^{\prime}-\frac{1}{2} \frac{\mathfrak{U}_{u}^{\prime \prime}}{\mathfrak{U}^{\prime \prime}}-\frac{1}{6} \frac{H_{u}}{H}, \\
& \text { (46) } \mathfrak{E}_{1}^{\prime}=\mathfrak{S}^{\prime}-A_{u}^{\prime}+B_{v}^{\prime}-\frac{1}{3}\left(\frac{\mathfrak{A}_{u v}^{\prime \prime}}{\mathfrak{U}^{\prime \prime}}+\frac{H_{u v}}{H}-\frac{\mathfrak{A}_{u}^{\prime \prime} \mathfrak{A}_{v}^{\prime \prime}}{\mathfrak{A}^{\prime \prime 2}}-\frac{H_{u} H_{v}}{H^{2}}\right) \text {, } \\
& \mathfrak{A}_{1}^{\prime \prime}=\frac{1}{H}\left(\mathfrak{A}^{\prime \prime} K+\mathfrak{S}_{v}^{\prime \prime}-\mathfrak{S}^{\prime \prime} \frac{\mathfrak{U}_{v}^{\prime \prime}}{\mathfrak{U}^{\prime \prime}}\right), \\
& \mathfrak{S}_{1}^{\prime \prime}=\mathfrak{S}^{\prime \prime}+3 A^{\prime}\left(\frac{\mathfrak{U}_{v}^{\prime \prime}}{\mathfrak{U}^{\prime \prime}}-\frac{H_{v}}{H}\right)+\frac{\mathfrak{A}_{v}^{\prime \prime}}{\mathfrak{A}^{\prime \prime}} \frac{H_{v}}{H}-3 A_{v}^{\prime}-\frac{H_{v v}}{H} .
\end{aligned}
$$

We find further

$$
H_{1}=2 H-K-\frac{\hat{o}^{2} \log H}{\partial u \partial v}, \quad K_{1}=H .
$$

We now proceed to set down the corresponding equations for the minus first 
Laplacian transformed net. We shall find

where

$$
\begin{aligned}
& \rho_{u u}=a_{-1} \rho_{u}+b_{-1} \rho_{v}+c_{-1} \rho, \\
& \rho_{u v}=a_{-1}^{\prime} \rho_{u}+b_{-1}^{\prime} \rho_{v}+c_{-1}^{\prime} \rho, \\
& \rho_{v v}=a_{-1}^{\prime \prime} \rho_{u}+b_{-1}^{\prime \prime} \rho_{v}+c_{-1}^{\prime \prime} \rho,
\end{aligned}
$$

$$
\begin{aligned}
& B K a_{-1}=\left(B_{u}-B B^{\prime}\right) K, \quad B K b_{-1}=B \mathfrak{E}_{u}-B_{u} \mathfrak{E}+B^{2} H, \\
& B K c_{-1}=B K \mathfrak{C}-A^{\prime}\left(B \mathfrak{C}_{u}-B_{u} \mathfrak{E}+B^{2} H\right)+2 K\left(B^{\prime} B_{u}-B B_{u}^{\prime}+B B^{\prime 2}\right), \\
& B K a_{-1}^{\prime}=A^{\prime} B K, \quad B K b_{-1}^{\prime}=B B^{\prime} K+B K_{u}, \\
& B K c_{-1}^{\prime}=-A^{\prime} B K_{u}+B K^{2}+B K\left(A_{u}^{\prime}-A^{\prime} B^{\prime}\right) \\
& B K a_{-1}^{\prime \prime}=K^{2}, \quad B K b_{-1}^{\prime \prime}=B_{v} K+B K_{v}-A^{\prime} B K \\
& B K c_{-1}^{\prime \prime}=-A^{\prime} B K_{v}+2 B^{\prime} K^{2}+K\left(B A_{v}^{\prime}-A^{\prime} B_{v}+2 A^{\prime 2} B\right)
\end{aligned}
$$

In order to reduce $(47)$ to its canonical form we put

$$
y_{-1}=\frac{\rho}{\sqrt[3]{B K}}
$$

The coefficients of the resulting system, the seminvariants of $(47)$, are :

$$
\begin{aligned}
& A_{-1}=-B^{\prime}+\frac{B_{u}}{B}-\frac{2}{3}\left(\frac{B_{u}}{B}+\frac{K_{u}}{K}\right), \quad B_{-1}=\frac{1}{B K}\left(B \mathfrak{S}_{u}-B_{u} \mathfrak{E}+B^{2} H\right), \\
& C_{-1}=\mathfrak{E}-\frac{A^{\prime}}{B K}\left(B \mathfrak{C}_{u}-B_{u} \mathfrak{E}+B^{2} H\right)+2\left(B^{\prime} \frac{B_{u}}{B}-B_{u}^{\prime}+{B^{\prime 2}}^{2}\right) \\
& +\frac{1}{3}\left(\frac{B_{u}}{B}-B^{\prime}\right)\left(\frac{B_{u}}{B}+\frac{K_{u}}{K}\right)+\frac{1}{3 B K}\left(\frac{B_{v}}{B}+\frac{K_{v}}{K}\right)\left(B \mathfrak{C}_{u}-B_{u} \mathfrak{\complement}+B^{2} H\right) \\
& -\frac{1}{3}\left(\frac{B_{u u}}{B}+\frac{K_{u u}}{K}-\frac{B_{u}^{2}}{B^{2}}-\frac{K_{u}^{2}}{K^{2}}\right)-\frac{1}{9}\left(\frac{B_{u}}{\dot{B}}+\frac{K_{u}}{K}\right)^{2}, \\
& A_{-1}^{\prime}=A^{\prime}-\frac{1}{3}\left(\frac{B_{v}}{B}+\frac{K_{v}}{K}\right), \quad B_{-1}^{\prime}=B^{\prime}+\frac{K_{u}}{K}-\frac{1}{3}\left(\frac{B_{u}}{B}+\frac{K_{u}}{K}\right), \\
& C_{-1}^{\prime}=C^{\prime}+A_{u}^{\prime}-B_{v}^{\prime}-A^{\prime} \frac{K_{u}}{K}+\frac{1}{3} A^{\prime}\left(\frac{B_{u}}{B}+\frac{K_{u}}{K}\right)+\frac{1}{3}\left(B^{\prime}+\frac{K_{u}}{K}\right)\left(\frac{B_{v}}{B}+\frac{K_{n}}{K}\right) \\
& -\frac{1}{3}\left(\frac{B_{u v}}{B}+\frac{K_{u v}}{K}-\frac{B_{u} B_{v}}{B^{2}}-\frac{K_{u} K_{v}}{K^{2}}\right)-\frac{1}{9}\left(\frac{B_{u}}{B}+\frac{K_{u}}{K}\right)\left(\frac{B_{v}}{B}+\frac{K_{v}}{K}\right), \\
& A_{-1}^{\prime \prime}=\frac{K}{B}, \quad B_{-1}^{\prime \prime}=-A^{\prime}+\frac{1}{3}\left(\frac{B_{v}}{B}+\frac{K_{v}}{K}\right) \text {, } \\
& C_{-1}^{\prime \prime}=A_{v}^{\prime}+2 A^{\prime 2}+\frac{2 B^{\prime} K}{B}+\frac{1}{3} \frac{K}{B}\left(\frac{B_{u}}{B}+\frac{K_{u}}{K}\right)-\frac{4}{3} A^{\prime}\left(\frac{B_{v}}{B}+\frac{K_{v}}{K}\right) \\
& +\frac{5}{9}\left(\frac{B_{v}}{B}+\frac{K_{v}}{K}\right)^{2}-\frac{2}{3} \frac{B_{v}}{B} \frac{K_{v}}{K}-\frac{1}{3}\left(\frac{B_{v v}}{B}+\frac{K_{v v}}{K}\right) \text {. }
\end{aligned}
$$


The seven fundamental invariants of the minus first Laplacian transformed net have the following values :

$$
\begin{aligned}
\mathfrak{B}_{-1} & =\frac{1}{\mathfrak{B K}}\left(\mathfrak{B C}_{u}-\mathfrak{B}_{u} \mathfrak{C}+\mathfrak{B}^{2} H\right), \\
\mathfrak{E}_{-1} & =\mathfrak{C}+3 B^{\prime}\left(\frac{\mathfrak{B}_{u}}{\mathfrak{B}}-\frac{K_{u}}{K}\right)+\frac{\mathfrak{B}_{u}}{\mathfrak{B}} \frac{K_{u}}{K}-3 B_{u}^{\prime}-\frac{K_{u u}}{K}, \\
(51) \quad \mathfrak{A}_{-1}^{\prime} & =A^{\prime}-\frac{1}{2} \frac{\mathfrak{B}_{v}}{\mathfrak{B}}-\frac{1}{6} \frac{K_{v}}{K}, \quad \mathfrak{B}_{-1}^{\prime}=B^{\prime}-\frac{1}{3} \frac{\mathfrak{B}_{u}}{\mathfrak{B}}+\frac{2}{3} \frac{K_{u}}{K}+\frac{1}{6 \mathfrak{B}_{-1}} \frac{\partial \mathfrak{B}_{-1}}{\partial u}, \\
\mathfrak{S}_{-1}^{\prime} & =\mathfrak{C}^{\prime}+A_{u}^{\prime}-B_{v}^{\prime}-\frac{1}{3}\left(\frac{\mathfrak{B}_{u v}}{\mathfrak{B}}+\frac{K_{u v}}{K}-\frac{\mathfrak{P}_{u} \mathfrak{B}_{v}}{\mathfrak{B}^{2}}-\frac{K_{u} K_{v}}{K^{2}}\right), \\
\mathfrak{A}_{-1}^{\prime \prime} & =\frac{K}{\mathfrak{B}}, \quad \mathfrak{S}_{-1}^{\prime \prime}=\frac{1}{\mathfrak{B}}\left(K_{u}+3 B^{\prime} K\right),
\end{aligned}
$$

and furthermore

$$
H_{-1}=K, \quad K_{-1}=2 K-H-\frac{\partial^{2} \log K}{\partial u \partial v} .
$$

It is easy to show that these two Laplacian transformations may be regarded as inverse to each other, a fact which has been made use of in our notation. In general, we shall therefore obtain an infinity of nets of plane curves by the repetition of one or the other of these transformations. It may happen however that a certain one of these nets degenerates, as is the case for instance if either $H$ or $R$ vanishes. In all such cases the general integral of the equation

$$
\frac{\partial^{2} y}{\partial u \partial v}=A^{\prime} \frac{\partial y}{\partial u}+B^{\prime} \frac{\partial y}{\partial v}+C^{\prime} y
$$

can be obtained by quadratures, and we are in possession of a very elegant analytical theory of this differential equation from this point of view, a theory originated by Laplace and further developed by Darboux. To Darboux is due also a geometrical interpretation of this theory which puts it into relation on the one hand with the theory of conjugate nets of curves on curved surfaces, and on the other with the theory of congruences of straight lines. But these geometric theories as developed by Darboux are not projective theories, since a simple equation of form (53) does not suffice to characterize a surface projectively. A purely projective theory may be obtained by adding a second partial differential equation of the second order to (53), and this is essentially what has been done by the present author in his general projective theory of surfaces and congruences.*

* E. J. Wilczynski. Projective Differential Geometry of Curved Surfaces. Five memoirs. These Transactions, 1907-1909. Compare also Sur la théorie générale des congruences, soon to be published by the Royal Belgian Academy. 
In the present paper we have found a different geometric interpretation for Darboux's theory, which seems to have escaped notice until now, and moreover since we are considering, not a single linear partial differential equation of the second order, but a system of three such equations, our theory is a purely projective one. Every one of Darboux's analytical theorems furnishes a preliminary basis for a series of deeper investigations concerning such systems of partial differential equations or, geometrically speaking, concerning nets of plane curves. Suppose, for instance, that equation (53) is of such a character that its general solution may be obtained by quadratures. It will contain a certain number of arbitrary functions. The question remains : how to determine these arbitrary functions so as to satisfy the remaining two equations of the system, and this reduces to a problem in ordinary differential equations.

As the point $y$ moves along a curve $u=$ const. or $v=$ const. of the original net, the corresponding point $y_{1}$ or $\sigma$ describes a curve $u=$ const. or $v=$ const. of the first Laplacian transformed net. Now the lines $y y_{1}$ are the tangents of the curves $u=$ const. Let us speak of those curves of the transformed nets which are described by $y_{1}$ when $y$ moves along one of the curves $u=$ const., or by $y_{2}$ when $y$ moves along one of the curves $v=$ const. as direct, and the other curves of their respective nets as indirect transforms of the curves of the original net. The lines which join the points of a curve of the original net to the corresponding points of its direct Laplacian transform will then be tangents of the former curve. The lines which join the points of a curve of the original net to the corresponding point of its indirect Laplacian transform are tangent to the latter.

This terminology is convenient in enabling us to state the following simple theorem. If a net of plane curves is obtained from another by a Laplacian transformation, its two families of curves are respectively the direet transforms of one and the indirect transforms of the other of the two families of the original net. That family of the transformed net, which corresponds indirectly to a family of the original net, can never be conıposed of straight lines.

In fact, if the curves $v=$ const. in the first Laplacian transformed net are straight lines, $B$, and therefore, according to (45), $H$ must vanish identically. But if $H=0$, the transformed net is degenerate.

In a net obtained by Laplacian transformation from another net, those curves which correspond directly to curves of the original net may be straight lines. They will be straight lines in the first transformed net if and only if

$$
\mathfrak{A}^{\prime 2} K+\mathfrak{U}^{\prime \prime} \mathfrak{C}_{v}^{\prime \prime}-\mathfrak{C}^{\prime \prime} \mathfrak{U}_{v}^{\prime \prime}=0,
$$

and for the minus first, if and only if

$$
\mathfrak{B}^{2} H+\mathfrak{B C}_{u}-\mathfrak{C F}_{u}=0 .
$$


§5. Determination of those nets whose Laplacian transforms are merely projections of the original net.

By means of the Laplace transformation two nets are brought into what we may call a point-to-point correspondence, those being corresponding points which, in the two nets, correspond to the same pair of values $(u, v)$. Our equations are such that both the original and the transformed nets are referred to the same independent variables. Consequently if this correspondence is to be equivalent to a projective transformation, the corresponding invariants must be equal for all values of $u$ and $v$. Since, moreover, the seminvariants are expressible in terms of the invariants and their derivatives, the conditions necessary and sufficient for projective equivalence of a net and of its first Laplacian transformed net are

$$
\begin{gathered}
B_{1}=B, \quad C_{1}=C, \quad A_{1}^{\prime}=A^{\prime}, \quad B_{1}^{\prime}=B^{\prime}, \quad C_{1}^{\prime}=C^{\prime}, \\
A_{1}^{\prime \prime}=A^{\prime \prime}, \quad C_{1}^{\prime \prime}=C^{\prime \prime} .
\end{gathered}
$$

According to (45) we find, therefore, the following conditions for $A, B, C$, etc.:

$$
\begin{aligned}
& \frac{A_{u}^{\prime \prime}}{A^{\prime \prime}}+\frac{H_{u}}{H}=0 \quad \frac{H}{A^{\prime \prime}}=B, \\
& B_{u}^{\prime}+2 B^{\prime 2}+2 \frac{A^{\prime} H}{A^{\prime \prime}}+\frac{1}{3} \frac{H}{A^{\prime \prime}}\left(\frac{A_{v}^{\prime \prime}}{A^{\prime \prime}}+\frac{H_{v}}{H}\right)-\frac{2}{3} A_{u}^{\prime \prime} H_{u}=C, \\
& \frac{2}{3} \frac{H_{v}}{H}-\frac{1}{3} \frac{A_{v}^{\prime \prime}}{A^{\prime \prime}}=0, \quad B_{v}^{\prime}-A_{u}^{\prime}-B^{\prime} \frac{H_{v}}{H}+\frac{1}{3} B^{\prime}\left(\frac{A_{v}^{\prime \prime}}{A^{\prime \prime}}+\frac{H_{v}}{H}\right)=0, \\
& \frac{A^{\prime \prime} K}{H}+\frac{1}{H}\left(\mathfrak{S}_{v}^{\prime \prime}-\mathfrak{S}^{\prime \prime} \frac{A_{v}^{\prime \prime}}{A^{\prime \prime}}\right)=A^{\prime \prime}, \\
& \mathfrak{S}^{\prime \prime}-\frac{B^{\prime}}{H}\left(\mathfrak{S}_{v}^{\prime \prime}-\mathfrak{S}^{\prime \prime} \frac{A_{v}^{\prime \prime}}{A^{\prime \prime}}+K A^{\prime \prime}\right)+2\left(A^{\prime} \frac{A_{v}^{\prime \prime}}{A^{\prime \prime}}-A_{v}^{\prime}+A^{\prime 2}\right) \\
& \quad+\frac{1}{3}\left(-A^{\prime}+\frac{A_{v}^{\prime \prime}}{A^{\prime \prime}}\right)\left(\frac{A_{v}^{\prime \prime}}{A^{\prime \prime}}+\frac{H_{v}}{H}\right)-\frac{1}{9}\left(\frac{A_{v}^{\prime \prime}}{A^{\prime \prime}}+\frac{H_{v}}{H}\right)^{2}-\frac{1}{3} \frac{\partial}{\partial v}\left(\frac{A_{v}^{\prime \prime}}{A^{\prime \prime}}+\frac{H_{v}}{H}\right)=C^{\prime \prime} .
\end{aligned}
$$

The first and fourth of these conditions give, upon integration,

$$
A^{\prime \prime} H=\psi(v), \quad A^{\prime \prime} H^{-2}=\phi(u),
$$

$\phi(u)$ and $\psi(v)$ being functions of the single arguments indicated.* Consequently

$$
A^{\prime \prime}=\phi^{\frac{1}{3}} \psi^{\frac{3}{3}}, \quad H=\phi^{-\frac{1}{3}} \psi^{\frac{1}{3}} .
$$

Now $A^{\prime \prime}$ and $H$ are relative invariants, and if we put

$$
\bar{u}=U(u), \quad \bar{v}=V(v),
$$

* Neither of these functions can vanish identically, since $A^{\prime \prime}$ and $H$ must be different from zero. Otherwise the transformed net would be degenerate. 
they are transformed into

$$
\bar{A}^{\prime \prime}=A^{\prime \prime} \frac{U^{\prime}}{\left(V^{\prime}\right)^{2}}, \quad \bar{H}=\frac{H}{U^{\prime}} \overline{V^{\prime}}
$$

respectively. We may, therefore, reduce them to unity by choosing $U^{\prime}$ and $V^{\prime}$ so that

$$
\left(U^{\prime}\right)^{3}=\frac{1}{\phi(u)}, \quad\left(V^{\prime}\right)^{3}=\psi(v) .
$$

The most general transformation of the form (56) which leaves the conditions $A^{\prime \prime}=H=1$ invariant will be conditioned by the equations

$$
\left(U^{\prime}\right)^{3}=1, \quad\left(V^{\prime}\right)^{3}=1 .
$$

The conditions (55) now reduce considerably. They become

$$
\begin{aligned}
& A^{\prime \prime}=1, \quad H=C^{\prime}+A^{\prime} B^{\prime}-A_{u}^{\prime}=1, \quad B=1, \\
& 2 A^{\prime}+B_{u}^{\prime}+2{B^{\prime}}^{2}=C, \quad B_{v}^{\prime}-A_{u}^{\prime}=0, \quad K+\mathfrak{C}_{v}^{\prime \prime}=1, \\
& \mathfrak{C}^{\prime \prime}-B^{\prime}+2\left(-A_{v}^{\prime}+A^{\prime 2}\right)=C^{\prime \prime} \text {, }
\end{aligned}
$$

whence

$$
\begin{gathered}
A^{\prime \prime}=1, \quad B=1, \quad A^{\prime}=\phi_{v}, \quad B^{\prime}=\phi_{u}, \quad C^{\prime}=1-\phi_{u} \phi_{v}+\phi_{u v}, \\
C=\phi_{u u}+2 \phi_{v}+2 \phi_{u}^{2}, \quad C^{\prime \prime}=\phi_{v v}+2 \phi_{v}^{2}-\phi_{u}+\lambda(u) .
\end{gathered}
$$

Here $\lambda(u)$ is an arbitrary function of $u$, and $\phi$ is subject to the equation $\phi_{v v}=0$, so that

$$
\phi=\mu(u)+\nu(u) v,
$$

$\mu(u), \nu(u)$ being arbitrary functions of $u$. Let us denote $d \mu / d u, d^{2} \mu / d u^{2}$ by $\mu^{\prime}, \mu^{\prime \prime}$, etc., then we shall have

$$
\begin{array}{lll}
A=-B^{\prime}, & B=1, & C=\mu^{\prime \prime}+2 \nu+2 \mu^{2}+\left(\nu^{\prime \prime}+4 \mu^{\prime} \nu^{\prime}\right) v+2\left(\nu^{\prime}\right)^{2} v^{2}, \\
A^{\prime}=\nu, & B^{\prime}=\mu^{\prime}+\nu^{\prime} v, & C^{\prime}=1-\nu \mu^{\prime}+\nu^{\prime \prime}-\nu \nu^{\prime} v, \\
A^{\prime \prime}=1, & B^{\prime \prime}=-A^{\prime}, & C^{\prime \prime}=\lambda+2 \nu^{2}-\mu^{\prime}-\nu^{\prime} v .
\end{array}
$$

These quantities must satisfy the integrability conditions (14). Imposing this condition we find that we must have

$$
\lambda=3 l, \quad \mu=k+l u, \quad \nu=m,
$$

where $k, l, m$ are arbitrary constants, so that we find finally

$$
\begin{aligned}
& A=-l, \quad B=1, \quad C=2\left(l^{2}+m\right), \\
& A^{\prime}=m, \quad B^{\prime}=l, \quad C^{\prime}=1-l m, \\
& A^{\prime \prime}=1, \quad B^{\prime \prime}=-m, \quad C^{\prime \prime}=2\left(l+m^{2}\right) \text {. }
\end{aligned}
$$

If a net is projectively equivalent to its first Laplacian transform, its system 
of differential equations can be reduced to one with the constant coefficients (57) which involve two arbitraries.

A system of partial differential equations of this form may be integrated as follows. The function

$$
y=e^{a u+\beta v}
$$

will be a solution of it, if $x$ and $\beta$ can be determined so as to satisfy the three conditions

$$
\begin{aligned}
\alpha^{2} & =-l \alpha+\beta+2\left(l^{2}+m\right), \\
\alpha \beta & =m \alpha+l \beta+1-l m, \\
\beta^{2} & =\alpha-m \beta+2\left(l+m^{2}\right),
\end{aligned}
$$

and these, it is easy to show, are consistent. The elimination of $\beta$ leads to the following equation for $\alpha$ :

$$
f(\alpha)=\alpha^{3}-3\left(l^{2}+m\right) \alpha+2 l^{3}+3 l m-1=0 .
$$

Let $\alpha_{1}, \alpha_{2}, \alpha_{3}$ be the three roots of this cubic, and let

$$
\beta_{k}=\frac{m \alpha_{k}+1-l m}{\alpha_{k}-l}
$$

be the corresponding values of $\beta$. In order that the three solutions of our system of partial differential equations

$$
y^{(k)}=e^{a_{k} u+\beta_{k} v} \quad(k=1,2,3),
$$

may be linearly independent it is necessary and sufficient that the three roots $\alpha_{k}$ of (59) be distinct, as may be seen by applying the usual test. Let us assume, therefore, that the discriminant of (59) or

$$
\Delta=1-6 l m-3 l^{2} m^{2}-4\left(l^{3}+m^{3}\right)
$$

is different from zero. Then the three quantities $\beta_{1}, \beta_{2}, \beta_{3}$, will also be distinct, and the net defined by equations (61) will be non-degenerate. In fact the determinant $D$ [cf. eq. (3)] becomes

$$
D=\left|\begin{array}{ccc}
1 & 1 & 1 \\
\alpha_{1} & \alpha_{2} & \alpha_{3} \\
\beta_{1} & \beta_{2} & \beta_{3}
\end{array}\right|=\Sigma\left(\alpha_{2} \beta_{3}-\alpha_{3} \beta_{2}\right) .
$$

On account of $(60)$ we may write

$$
\begin{aligned}
D & =\Sigma \frac{\left(\alpha_{2}-\alpha_{3}\right)\left[m \alpha_{2} \alpha_{3}+(1-l m)\left(\alpha_{2}+\alpha_{3}\right)-l(1-l m)\right]}{\left(\alpha_{2}-l\right)\left(\alpha_{3}-l\right)} \\
& =\Sigma \frac{\left(\alpha_{2}-\alpha_{3}\right)\left[m \alpha_{2} \alpha_{3}+(1-l m)\left(\alpha_{2}+\alpha_{3}\right)-l(1-l m)\right]}{\left(\alpha_{1}-l\right)\left(\alpha_{2}-l\right)\left(\alpha_{3}-l\right)} . l
\end{aligned}
$$


The denominator of this fraction is equal to

Consequently

$$
-f(l)=1
$$

$$
D=\Sigma\left(\alpha_{2}-\alpha_{3}\right)\left[m \alpha_{1} \alpha_{2} \alpha_{3}+(1-l m)\left(\alpha_{1} \alpha_{2}+\alpha_{1} \alpha_{3}\right)-l(1-l m) \alpha_{1}\right.
$$

But, according to (59),

$$
\left.-l m \alpha_{2} \alpha_{3}-l(1-l m)\left(\alpha_{2}+\alpha_{3}\right)+l^{2}(1-l m)\right] \text {. }
$$

$$
\alpha_{1}+\alpha_{2}+\alpha_{3}=0, \quad \alpha_{2} \alpha_{3}+\alpha_{3} \alpha_{1}+\alpha_{1} \alpha_{2}=-3\left(l^{2}+m\right),
$$

Consequently

$$
\alpha_{1} \alpha_{2} \alpha_{3}=1-3 l m-2 l^{3} \text {. }
$$

$$
\begin{aligned}
D & =\Sigma\left(\alpha_{2}-\alpha_{3}\right)\left[m\left(1-3 l m-2 l^{3}\right)-3\left(l^{2}+m\right)(1-l m)+l^{2}(1-l m)-\alpha_{2} \alpha_{3}\right] \\
& =\left[m\left(1-3 l m-2 l^{3}\right)-3\left(l^{2}+m\right)(1-l m)+l^{2}(1-l m)\right] \Sigma\left(\alpha_{2}-\alpha_{3}\right)-\Sigma\left(\alpha_{2}^{2} \alpha_{3}-\alpha_{2} \alpha_{3}^{2}\right) .
\end{aligned}
$$

But obviously

so that

$$
\Sigma\left(\alpha_{2}-\alpha_{3}\right)=0, \quad \Sigma\left(\alpha_{2}^{2} \alpha_{3}-\alpha_{2} \alpha_{3}^{2}\right)=-\Delta
$$

$$
D=\Delta
$$

and is consequently different from zero. Therefore equations (61) define a non-degenerate net of plane curves if the quantities $\alpha_{1}, \alpha_{2}, \alpha_{3}$ are distinct.

We proceed to discuss the case when two of the roots of (59) are equal, say

$$
\alpha_{3}=\alpha_{2}, \quad \Delta=0,
$$

while $\alpha_{1}$ and $\alpha_{2}$ are distinct. We may put again

$$
y^{(k)}=e^{a_{k} u+\beta_{k} v} \quad(k=1,2),
$$

but it remains to find a third solution of our system of partial differential equations. The second equation of (58) may be put into the form

so that we may write

$$
(\alpha-l)(\beta-m)=1 \text {, }
$$

and consider the cubic

$$
\alpha-l=t, \quad \beta-m=\frac{1}{t},
$$

$$
t^{3}+3 l t^{2}-3 m t-1=0
$$

instead of (59). If we denote its roots by $t_{1}, t_{2}, t_{3}$ we have in our case $t_{3}=t_{2}$. According to well-known principles, the function obtained from

$$
\frac{\partial}{\partial t}\left[e^{(l+t) u+(m+1 / t) v}\right]
$$

by substituting $t=t_{2}$ after the differentiation, will be a solution of our partial differential equations if $t_{2}$ is a double root of (65). We thus find

$$
y^{(3)}=\left[\left(\alpha_{2}-l\right) u-\left(\beta_{2}-m\right) v\right] e^{\alpha_{2} u+\beta_{2} v} \text {. }
$$


That this function satisfies the differential equations may also be verified directly. The linear independence of the three functions $y^{(k)}$ and the non-degeneracy of the net may be verified as before, or even more simply as follows. We find

whence

$$
\begin{gathered}
\frac{y^{(3)}}{y^{(2)}}=\left(\alpha_{2}-l\right) u-\left(\beta_{2}-m\right) v, \\
\log \frac{y^{(1)}}{y^{(2)}}=\left(x_{1}-\alpha_{2}\right) u+\left(\beta_{1}-\beta_{2}\right) v,
\end{gathered}
$$

$$
\begin{array}{r}
t_{1} t_{2}^{2} \log \frac{y^{(1)}}{y^{(2)}}+t_{1} t_{2}\left(t_{2}-t_{1}\right) \frac{y^{(3)}}{y^{(2)}}=\left(t_{1}-t_{2}\right)^{2} v, \\
t_{1} \log \frac{y^{(1)}}{y^{(2)}}+\left(t_{2}-t_{1}\right) \frac{y^{(3)}}{y^{(2)}}=\left(t_{1}-t_{2}\right)^{2} u,
\end{array}
$$

as the finite equations of the curves of our net. We see that neither family of curves degenerates, since $t_{2} \neq t_{1}$. Moreover, the three functions are linearly independent, since no root of (65) can be equal to zero, and consequently the curves (67) cannot reduce to straight lines.

If all three roots of (59) are equal, their common value must be zero, and we must have

so that either

$$
l^{2}+m=0, \quad 1-3 l m-2 l^{3}=0,
$$

$l=-1, \quad m=-1 \quad$ or $\quad l=-\omega, \quad m=-\omega^{2} \quad$ or $\quad l=-\omega^{2}, \quad m=-\omega$, where $\omega$ is a primitive third root of unity. If we write down the three systems of differential equations which correspond to these three alternatives, we find that they may be transformed into each other by a transformation of the form

$$
\bar{u}=\alpha u, \quad \bar{v}=\beta v,
$$

where $\alpha$ and $\beta$ are constants. It suffices therefore to consider the case

$$
l=m=-1 \text {, }
$$

which gives rise to the following system of differential equations :

$$
y_{u u}=y_{u}+y_{v}, \quad y_{u v}=-y_{u}-y_{n}, \quad y_{v v}=y_{u}+y_{v} .
$$

This system has the following three linearly independent solutions;

$$
y^{(1)}=(u-v)^{2}+u+v, \quad y^{(2)}=u-v, \quad y^{(3)}=1 .
$$

Consequently the equations of the two one-parameter families of the net will be

$$
\begin{aligned}
& \left(x_{1}+x_{2}\right) x_{3}-x_{2}^{2}-2 u x_{3}^{2}=0 \\
& \left(x_{1}-x_{2}\right) x_{3}-x_{2}^{2}-2 v x_{3}^{2}=0 ;
\end{aligned}
$$


i. e., both families are pencils of conics, which may be completely characterized as follows. The conics $u=$ const. all have four-pointic (third order) contact with

$$
\left(x_{1}+x_{2}\right) x_{3}-x_{2}^{2}=0
$$

at the vertex $A\left(x_{2}=x_{3}=0\right)$ of the triangle of reference. Similarly, the conics $v=$ const. have four-pointic contact with the conic

$$
\left(x_{1}-x_{2}\right) x_{3}-x_{2}^{2}=0
$$

at the point $A$. It remains to characterize the two conics (69) and (70). The conic (69) touches the line $x_{1}+x_{2}=0$ at the vertex $C\left(x_{1}=x_{2}=0\right)$ of the fundamental triangle, while (70) at the same point touches the line $x_{1}-x_{2}=0$, and these two lines divide harmonically the sides of the triangle $A C$ and $B C$ which meet in $C$. The third side of the triangle $B C\left(x_{1}=0\right)$ meets the two conics in two points which divide it harmonically. We may, therefore, construct our two families of conics as follows.

Choose an arbitrary triangle and denote its vertices by $A, B, C$. Through the vertex $C$ draw two lines, $l$ and $m$, which divide $C A$ and $C B$ harmonically. On the side $B C$ of the triangle choose any two points, $D$ and $E$, which divide $B C$ harmonically. Construct the conic $K_{1}$ tangent to $A B$ at $A$, tangent to $l$ at $C$ and passing through $D$. Construct also the conic $K_{2}$, tangent to $A B$ at $A$, tangent to $m$ at $C$, and passing through $E$. The two pencils of conics, which have four-pointic contact with $K_{1}$ and $K_{2}$ respectively at $A$, determine the most general net of conics which has the property that its Laplacian transformed nets are projectively equivalent to it.

Let us return to the general case, in which the three numbers $\alpha_{1}, \alpha_{2}, \alpha_{3}$ are distinct. The curves of the net are, of course, anharmonic curves, using Halphen's terminology, since the linear differential equations of the third order which define them have constant coefficients.* For every curve of this kind there exists a one-parameter group of collineations which converts it into itself, and in the general case this collineation group has an invariant triangle. From this remark it easily follows that the three points in which a tangent of the curve intersects the sides of the invariant triangle, together with its point of contact, will determine an anharmonic ratio, constant along the whole curve. This constant anharmonic ratio is an absolute invariant of the curve, but its expression in terms of the coefficients of the differential equation is irrational. In fact, the six values of this anharmonic ratio, which correspond to the different possible arrangements of the four points on the tangent, are the roots of the following equation of the sixth order

$$
3^{9} \frac{\left(\lambda^{2}-\lambda+1\right)^{3}}{(\lambda-2)^{2}(1-2 \lambda)^{2}(\lambda+1)^{2}}=\frac{\theta_{8}^{3}}{\theta_{3}^{8}},
$$

* Cf. equations (73) to (76) of the following paragraph. See also E. J. WilczYnski, Projective Differential Geometry, pp. 86-9C. 
where

$$
\frac{\theta_{8}^{3}}{\theta_{3}^{8}}=\frac{3^{9}\left(m+l^{2}\right)^{3}}{\left(2 l^{3}+3 l m-1\right)^{2}}
$$

for the curves $v=$ const. and

for the curves $u=$ const.*

$$
\frac{\theta_{8}^{3}}{\theta_{3}^{8}}=\frac{3^{9}\left(l+m^{2}\right)^{3}}{\left(2 m^{3}+3 l m-1\right)^{2}}
$$

It is not difficult to show that, when the invariant triangle and the value $K$ of the anharmonic ratio which corresponds to a definite arrangement of the four points on the tangent are given, there exists a unique one-parameter family of anharmonic curves belonging to the given triangle and the given anharmonic ratio in the sense of the above theorem. This being so, we can describe our net in the general case [all roots of (59) distinct] as follows.

Construct an arbitrary triangle. Let $\lambda$ and $\lambda^{\prime}$ be two numbers different from each other, from zero, from $\pm 1,2$ or $\frac{1}{2} \cdot \dagger$ Construct the two one-parameter families of anharmonic curves which have the given triangle as invariant triangle, and whose curves belong to the anharmonic ratios $\lambda$ and $\lambda^{\prime}$ respectively. Any net constructed in this way will be projectively equivalent with those obtained from it by Laplacian transformations.

The assumption that $\lambda$ and $\lambda^{\prime}$ shall be distinct does not imply that the invariants (72a) and (72b) shall have different values. In fact, for a given value of the absolute invariant $\theta_{8}^{3} / \theta_{3}^{(8)},(71)$ shows that there are six values of $\lambda$ no two of which coincide, except in the cases which have been excluded.

The nature of the net in the intermediate case, when two roots of (59) coincide, but not all three, is most easily investigated as follows. Let

$$
\frac{y^{(2)}}{y^{(3)}}=x, \quad \frac{y^{(1)}}{y^{(3)}}=y
$$

be cartesian coördinates. Then equations (67) may be written

where

$$
t_{2} \log y+\left(t_{2}-t_{1}\right) x=\bar{v}, \quad t_{1} \log y+\left(t_{2}-t_{1}\right) x=\bar{u},
$$

or, more simply,

$$
\bar{v}=\frac{\left(t_{1}-t_{2}\right)^{2}}{t_{1} t_{2}} v, \quad \bar{u}=\left(t_{1}-t_{2}\right)^{2} u
$$

where

$$
y=U e^{\frac{\left(t_{1}-t_{2}\right) x}{t_{1}}}, \quad y=V e^{\frac{\left(t_{1}-t_{2}\right) x}{t_{2}}},
$$

$$
U=e^{\frac{\pi}{t_{1}}}, \quad V=e^{\frac{\nabla}{t_{2}}}
$$

* Loo. cit., pp. (58), (59), (88).

† These values are excluded because they correspond to the cases in which the three quantities $\alpha_{1}, \alpha_{2}, \alpha_{3}$ are not all distinct. 
so that the families $u=$ const., $v=$ const., coincide with the families $U=$ const., $V=$ const., respectively.

In our case $t_{1}$ and $t_{2}$ are distinct, so that the equations may be written

$$
y=U A^{x}, \quad y=V B^{x},
$$

where $A$ and $B$ are two distinct numbers, which may be thought of as being given arbitrarily.

We therefore obtain a net of the desired character if we construct two exponential curves

$$
y=A^{x}, \quad y=B^{x},
$$

with arbitrary, but distinct bases, and then magnify their ordinates in all possible ratios. The most general net of the kind considered is obtained from this one by projective transformation.

It is of interest to notice the further result that the system of differential equations with constant coefficients which has presented itself in this theory is the most general completely integrable system of form (4) whose invariants are constants. This may be verified easily by making use of the integrability conditions in the form (14).

\section{$\S 6$. Osculating conics of curves of the net.}

An individual plane curve is characterized projectively by a linear differential equation of the third order. We now proceed to determine these equations for the curves $u=$ const. and $v=$ const. of a net when the latter is defined by a system of form (4).

We have

$$
y_{u u}=a y_{u}+b y_{v}+c y,
$$

and consequently by differentiation and making use of (4)

$$
y_{u u u}=\left(a_{u}+a^{2}+a^{\prime} b+c\right) y_{u}+\left(b_{u}+a b+b b^{\prime}\right) y_{v}+\left(c_{u}+a c+b c^{\prime}\right) y \text {. }
$$

The elimination of $y_{v}$ between these two equations gives the desired equation for the curves $v=$ const., viz.,

$$
y_{u u u}+3 p_{1} y_{u u}+3 p_{2} y_{u}+p_{3} y=0,
$$

where

$$
\begin{gathered}
p_{1}=-\frac{1}{3}\left(\frac{b_{u}}{b}+a+b^{\prime}\right), \quad p_{2}=-\frac{1}{3}\left(a_{u}-a \frac{b_{u}}{b}+c+a^{\prime} b-a b^{\prime}\right), \\
p_{3}=-\left(c_{u}-c \frac{b_{u}}{b}+b c^{\prime}-b^{\prime} c\right) .
\end{gathered}
$$

Similarly we find the differential equations of the curves $u=$ const., viz.,

$$
y_{v v v}+3 q_{1} y_{v v}+3 q_{2} y_{v}+q_{3} y=0
$$


where

$$
\begin{gathered}
q_{1}=-\frac{1}{3}\left(\frac{a_{v}^{\prime \prime}}{a^{\prime \prime}}+a^{\prime}+b^{\prime \prime}\right), \quad q_{2}=-\frac{1}{3}\left(b_{v}^{\prime \prime}-b^{\prime \prime} \frac{a_{v}^{\prime \prime}}{a^{\prime \prime}}+c^{\prime \prime}+a^{\prime \prime} b^{\prime}-a^{\prime} b^{\prime \prime}\right) \\
q_{3}=-\left(c_{v}^{\prime \prime}-c^{\prime \prime} \frac{a_{v}^{\prime \prime}}{a^{\prime \prime}}+a^{\prime \prime} c^{\prime}-a^{\prime} c^{\prime \prime}\right) .
\end{gathered}
$$

The seminvariants and semi-covariants of $(73)$ are *

$$
\begin{array}{ll}
P_{2}=p_{2}-p_{1}^{2}-\frac{\partial p_{1}}{\partial u}, & P_{3}=p_{3}-3 p_{1} p_{2}+2 p_{1}^{3}-\frac{\partial^{2} p_{1}}{\partial u^{2}}, \\
z^{(u)}=y_{u}+p_{1} y, & \rho^{(u)}=y_{u u}+2 p_{1} y_{u}+p_{2} y
\end{array}
$$

the corresponding functions for (75) are

$$
\begin{array}{ll}
Q_{2}=q_{2}-q_{1}^{2}-\frac{\partial q_{1}}{\partial v}, & Q_{3}=q_{3}-3 q_{1} q_{2}+2 q_{1}^{3}-\frac{\partial^{2} q_{1}}{\partial v^{2}}, \\
z^{(v)}=y_{v}+q_{1} y, & \rho^{(v)}=y_{v o}+2 q_{1} y_{v}+q_{2} y .
\end{array}
$$

If we substitute into the expressions for $z^{(u)}, y=y^{(k)}(k=1,2,3)$, where $y^{(1)}, y^{(2)}, y^{(3)}$ are three linearly independent solutions of system (4), $z^{(u)}$ assumes three values which we interpret as the homogeneous coördinates of a point. This point $z^{(u)}$ will obviously be on the tangent of the curve $v=$ const. constructed at the point $y$. Similarly $\rho^{(u)}$ represents a third point of the plane, and these three points will not be collinear unless $y$ is a point of inflection of the curve $v=$ const. under consideration. Any expression of the form

$$
a_{1} y+a_{2} z^{(u)}+a_{3} \rho^{(u)}
$$

will then represent a point, whose coördinates, referred to the triangle $y, z^{(u)}, \rho^{(u)}$ may be chosen proportional to $a_{1}, a_{2}, a_{3}$ respectively. We shall prefer, however, to use the notation

$$
a_{k}=x_{k}^{(u)} \quad(k=1,2,3) .
$$

Referred to this system of coördinates, the equation of the conic having fourthorder contact with the curve $v=$ const. at $y$ is

$$
x_{2}^{(u)_{2}}-2 x_{1}^{(u)} x_{3}^{(u)}+P_{2} x_{3}^{(u)_{2}}=0 . \dagger
$$

We wish to find the equation of this same conic referred to the triangle of reference $y, z, \rho$ whose geometrical significance in connection with a given net has already been discussed. In order to do this we must find the equations of transformation between the two systems of coördinates.

Let $x_{1}, x_{2}, x_{3}$ be the coördinates of a point with respect to the triangle $y, \rho$,

* E. J. Wilczy nski, Projective Differential Geometry, pp. 58, 59.

† Ibid., p. 65. 
$\sigma$, and let $x_{1}^{(u)}, x_{2}^{(u)}, x_{3}^{(u)}$ be the coördinates of the same point with respect to the triangle $y, z^{(u)}, \rho^{(u)}$. Then we must have

$$
x_{1} y+x_{2} \rho+x_{3} \sigma=\omega\left(x_{1}^{(u)} y+x_{2}^{(u)} z^{(u)}+x_{3}^{(u)} \rho^{(u)}\right),
$$

where $\omega$ is a factor of proportionality. Now we find from the definition of these quantities the following relations:

$$
\begin{aligned}
& \rho=-\left(p_{1}+b^{\prime}\right) y+z^{(n)} \\
& \sigma=\frac{-a^{\prime} b-c+a p_{1}+2 p_{1}^{2}-p_{2}}{b} y-\frac{a+2 p_{1}}{b} z^{(u)}+\frac{1}{b} \rho^{(u)} .
\end{aligned}
$$

Let these values be substituted into the preceding equation. The coefficients of $y, z^{(u)}, \rho^{(u)}$ on both sides of the equation must be equal; otherwise we should find a linear homogeneous differential equation of the form

$$
L y_{u u}+P y_{u}+Q y=0
$$

satisfied by $y^{(1)}, y^{(2)}, y^{(3)}$; i. e., the curve $v=$ const. considered would be a straight line, which case we shall exclude. The exceptional case suggested by $(80)$, viz., $b=0$, has the same significance. Obviously in this exceptional case there is no proper osculating conic.

We obtain, therefore, the following equations of transformation

$$
\begin{aligned}
& \omega x_{1}^{(u)}=x_{1}-\left(p_{1}+b^{\prime}\right) x_{2}+\frac{1}{b}\left(-a^{\prime} b-c+a p_{1}+2 p_{1}^{2}-p_{2}\right) x_{3}, \\
& \omega x_{2}^{(u)}=x_{2}-\frac{a+2 p_{1}}{b} x_{3}, \\
& \omega x_{3}^{(u)}=\frac{1}{b} x_{3} .
\end{aligned}
$$

The equation of the conic (79) referred to the new triangle of reference becomes therefore

$$
\begin{aligned}
& b^{2} x_{2}^{2}+\frac{1}{3} b\left(2 \frac{b_{u}}{b}-4 a+8 b^{\prime}\right) x_{2} x_{3}-2 b x_{1} x_{3} \\
& \quad+\left(3 p_{2}-p_{1}^{2}-\frac{\partial p_{1}}{\partial u}+2 a p_{1}+a^{2}+2 a^{\prime} b+2 c\right) x_{3}^{2}=0 .
\end{aligned}
$$

Since our triangle of reference is a covariant triangle, the coefficients of this equation must be invariants of the net. In fact we find that equation (82) may be reduced to the following:

$$
M \equiv \mathfrak{V}^{2} x_{2}^{2}+4 \mathfrak{B} \mathfrak{B}^{\prime} x_{2} x_{3}-2 \mathfrak{R} x_{1} x_{3}+\phi x_{3}^{2}=0,
$$


where

$$
\phi=\mathfrak{E}-4 \mathfrak{B}^{\prime 2}+2 \mathfrak{B}_{u}^{\prime}+6 \mathfrak{B}^{\prime} B^{\prime} .
$$

In order to verify this statement, it is convenient to assume that system (4) is given in its canonical form, and to introduce for $p_{1}$ and $p_{2}$ their values from (74).

In the same way we may find the equation of the conic which osculates the curve $u=$ const. at the point $y$. We have, in this case, the relations

$$
\begin{aligned}
& \rho=\frac{1}{a^{\prime \prime}}\left[\left(-a^{\prime \prime} b^{\prime}-c^{\prime \prime}+b^{\prime \prime} q_{1}+2 q_{1}^{2}-q_{2}\right) y-\left(2 q_{1}+b^{\prime \prime}\right) z^{(v)}+\rho^{(v)}\right], \\
& \sigma=-\left(a^{\prime}+q_{1}\right) y+z^{(v)}
\end{aligned}
$$

so that

$$
\begin{aligned}
& \omega x_{1}^{(v)}=x_{1}+\frac{1}{a^{\prime \prime}}\left(-a^{\prime \prime} b^{\prime}-c^{\prime \prime}+b^{\prime \prime} q_{1}+2 q_{1}^{2}-q_{2}\right) x_{2}-\left(a^{\prime}+q_{1}\right) x_{3}, \\
& \omega x_{2}^{(v)}=-\frac{1}{a^{\prime \prime}}\left(2 q_{1}+b^{\prime \prime}\right) x_{2}+x_{3}, \\
& \omega x_{3}^{(v)}=\frac{1}{a^{\prime \prime}} x_{2} .
\end{aligned}
$$

The equation of the conic which osculates the curve $u=$ const. at the point $y$ becomes

$$
N \equiv \psi x_{2}^{2}+4 \mathfrak{U}^{\prime} \mathfrak{U}^{\prime \prime} x_{2} x_{3}+\mathfrak{U}^{\prime 2} x_{3}^{2}-2 \mathfrak{U}^{\prime \prime} x_{1} x_{2}=0
$$

where

$$
\psi=\mathfrak{C}^{\prime \prime}-4 \mathfrak{U}^{\prime 2}+2 \mathfrak{U}_{v}^{\prime}+6 \mathfrak{U}^{\prime} A^{\prime}
$$

Equations (83) and (87) enable us to interpret geometrically a number of invariants.

The line $x_{1}=0$, i. e., the line which joins the points $P_{\rho}$ and $P_{\sigma}$, will intersect the conic $M=0$ in two points which divide $P_{\rho} P_{\sigma}$ harmonically if and only if $\mathfrak{S}^{\prime}$ is equal to zero. Similarly the two points determined on $P_{\rho} P_{\sigma}$ by the conic $N=0$ divide $P_{\rho} P_{\sigma}$ harmonically if and only if $\mathfrak{A}^{\prime}=0$. The anharmonic ratios which these two groups of four points determine, if $\mathfrak{A}^{\prime}$ and $\mathfrak{C}^{\prime}$ are not zero, are easily expressible as absolute invariants. The point $P_{\sigma}$ is on the conic $M=0$ if and only if $\phi=0$; similarly $\psi$ will vanish if and only if $P_{\rho}$ is on the conic $N=0$.

Neither of the osculating conics is degenerate unless either $\mathfrak{B}$ or $\mathfrak{U}^{\prime \prime}$ vanishes, i. e., unless the point of osculation is a point of inflection of the corresponding curve $u=$ const. or $v=$ const.

The line $P_{\rho} P_{\sigma}$ is tangent to $M=0$ if and only if

$$
\phi-4 \mathfrak{B}^{\prime 2}=0
$$


it is tangent to $N=0$ if and only if

$$
\psi-4 \mathfrak{U}^{2}=0
$$

The conics $M=0$ and $N=0$, of course, have the point $P_{y}$ in common. The discriminant of the general conic

$$
\lambda M+\mu N=0
$$

of their pencil may be reduced to

$$
\mathfrak{B}^{4} \lambda^{3}+\mathfrak{B}^{2}\left(\Psi-4 \mathfrak{U}^{\prime \prime} \mathfrak{B}^{\prime}\right) \lambda^{2} \mu+\mathfrak{A}^{\prime 2}\left(\phi-4 \mathfrak{A}^{\prime} \mathfrak{B}\right) \lambda \mu^{2}+\mathfrak{A}^{\prime \prime 4} \mu^{3},
$$

and the values of $\lambda: \mu$ which annul this expression will determine the degenerate conics of the pencil, and consequently the remaining three points of intersection of $M=0$ and $N=0$. The two conics will touch each other if the discriminant of $(90)$ vanishes.

We have found the equations of the osculating conics of the curves of the net which meet at the point $P_{y}$ corresponding to the values $u$ and $v$ of the independent variables. We proceed to determine the osculating conics of the curves of the net in the vicinity of $P_{y}$.

Let us denote by $y^{\prime}, \rho^{\prime}, \sigma^{\prime}$ the vertices of the triangle which is obtained from $y, \rho, \sigma$ when the infinitesimal increments $\delta u$ and $\delta v$ are given to $u$ and $v$ respectively. We shall have

$$
\begin{aligned}
& y^{\prime}=y+y_{u} \delta u+y_{v} \delta v, \\
& \rho^{\prime}=\rho+\rho_{u} \delta u+\rho_{v} \delta v, \\
& \sigma^{\prime}=\sigma+\sigma_{u} \delta u+\sigma_{v} \delta v,
\end{aligned}
$$

retaining only terms of the first order in $\delta u$ and $\delta v$. Now let us assume that (4) is given in its canonical form. Then we find

$$
\begin{aligned}
y_{u} & =B^{\prime} y+\rho, \quad y_{v}=A^{\prime} y+\sigma, \\
y_{u u} & =\left(C-B^{\prime 2}+A^{\prime} B\right) y-B^{\prime} \rho+B \sigma, \\
y_{u v} & =\left(C^{\prime}+2 A^{\prime} B^{\prime}\right) y+A^{\prime} \rho+B^{\prime} \sigma, \\
y_{v v} & =\left(C^{\prime \prime}-A^{\prime 2}+A^{\prime \prime} B^{\prime}\right) y+A^{\prime \prime} \rho-A^{\prime} \sigma ;
\end{aligned}
$$

whence

$$
\begin{aligned}
& \rho_{u}=\mathfrak{\complement} y-2 B^{\prime} \rho+B \sigma, \\
& \rho_{v}=K^{\prime} y+A^{\prime} \rho, \\
& \sigma_{u}=H y+B^{\prime} \sigma, \\
& \sigma_{v}=\mathfrak{C}^{\prime \prime} y+A^{\prime \prime} \rho-2 A^{\prime} \sigma .
\end{aligned}
$$

The first and last of these equations provide us with geometrical interpretations for the invariant equations $\mathfrak{S}=0$ and $\mathfrak{S}^{\prime \prime}=0$ respectively. 
If $\mathbb{E}=0$, the curve described by $P_{p}$ as $P_{y}$ moves along a curve $v=$ const. is such that its tangent at $P_{\rho}$ passes through $P_{\sigma}$.

If $\mathfrak{S}^{\prime \prime}=\cdot 0$, the curve described by $P_{\sigma}$ as $P_{y}$ moves along a curve $u=$ const. is such that its tangent at $P_{\sigma}$ passes through $P_{\rho}$.

We substitute the values just found for $y_{u}, \ldots, \sigma_{v}$ into (91), and obtain

$$
\begin{aligned}
& y^{\prime}=y\left(1+B^{\prime} \delta u+A^{\prime} \delta v\right)+\rho \delta u+\sigma \delta v, \\
& \rho^{\prime}=y(\mathfrak{c} \delta u+K \delta v)+\rho\left(1-2 B^{\prime} \delta u+A^{\prime} \delta v\right)+\sigma B \delta u \text {, } \\
& \sigma^{\prime}=y\left(H \delta u+\mathbb{C}^{\prime \prime} \delta v\right)+\rho A^{\prime \prime} \delta v+\sigma\left(1+B^{\prime} \delta u-2 A^{\prime} \delta v\right) \text {. }
\end{aligned}
$$

Now let $x_{1}^{\prime}, x_{2}^{\prime}, x_{3}^{\prime}$ be the coördinates of a point with respect to the triangle $y^{\prime}, \rho^{\prime}, \sigma^{\prime}$, while $x_{1}, x_{2}, x_{3}$ are the coördinates of the same point with respect to $y$, $\rho, \sigma$. Then we shall have

whence

$$
\omega\left(x_{1} y+x_{2} \rho+x_{3} \sigma\right)=x_{1}^{\prime} y^{\prime}+x_{2}^{\prime} \rho^{\prime}+x_{3}^{\prime} \sigma^{\prime},
$$

$$
\begin{aligned}
& \omega x_{1}=\left(1+B^{\prime} \delta u+A^{\prime} \delta v\right) x_{1}^{\prime}+\left((5 \delta u+K \delta v) x_{2}^{\prime}+\left(H \delta u+\mathcal{S}^{\prime \prime} \delta v\right) x_{3}^{\prime},\right. \\
& \omega x_{2}=\delta u \cdot x_{1}^{\prime}+\left(1-2 B^{\prime} \delta u+A^{\prime} \delta v\right) x_{2}^{\prime}+A^{\prime \prime} \delta v \cdot x_{3}^{\prime}, \\
& \omega x_{3}=\delta v \cdot x_{1}^{\prime}+B \delta u \cdot x_{2}^{\prime}+\left(1+B^{\prime} \delta u-2 A^{\prime} \delta v\right) x_{3}^{\prime},
\end{aligned}
$$

or inversely

$$
\begin{aligned}
& \omega^{\prime} x_{1}^{\prime}=x_{1}-\left(B^{\prime} x_{1}+\mathcal{E} x_{2}+H x_{3}\right) \delta u-\left(A^{\prime} x_{1}+K x_{2}+\mathcal{C}^{\prime \prime} x_{3}\right) \delta v, \\
& \omega^{\prime} x_{2}^{\prime}=x_{2}-\left(x_{1}-2 B^{\prime} x_{2}\right) \delta u-\left(A^{\prime} x_{2}+A^{\prime \prime} x_{3}\right) \delta v, \\
& \omega^{\prime} x_{3}^{\prime}=x_{3}-\left(B x_{2}+B^{\prime} x_{3}\right) \delta u-\left(x_{1}-2 A^{\prime} x_{3}\right) \delta v .
\end{aligned}
$$

The equation of the conic which osculates the curve $v=$ const. of our net at the point $(u+\delta u, v+\delta v)$, referred to the triangle $y^{\prime}, \rho^{\prime}, \sigma^{\prime}$ will be

$$
\begin{aligned}
\left(\mathfrak{B}^{2}+2 \mathfrak{B P}_{u} \delta u+2 \mathfrak{B P}_{v} \delta v\right){x_{2}^{\prime}}_{2}^{2}+4\left(\mathfrak{B P}^{\prime}+\frac{\partial\left(\mathfrak{B} \mathfrak{B}^{\prime}\right)}{\partial u} \delta u+\frac{\partial\left(\mathfrak{B P}^{\prime}\right)}{\partial v} \delta v\right) x_{2}^{\prime} x_{3}^{\prime} \\
-2\left(\mathfrak{B}+\mathfrak{B}_{u} \delta u+\mathfrak{B}_{v} \delta v\right) x_{1}^{\prime} x_{3}^{\prime}+\left(\phi+\phi_{u} \delta u+\phi_{v} \delta v\right) x_{3}^{\prime 2}=0,
\end{aligned}
$$

If we introduce the values $(96)$ of $x_{1}^{\prime}, x_{2}^{\prime}, x_{3}^{\prime}$ this equation may be reduced to

$$
\left(1+\frac{4}{3} \frac{B_{u}}{B} \delta u\right) M+\left(\phi_{u}-2 B^{\prime} \phi-\frac{4}{3} \frac{\mathfrak{B}_{n}}{\mathfrak{B}} \phi+2 \mathfrak{B} H\right) x_{3}^{2} \delta u+P \delta v=0,
$$

where

$$
\begin{array}{rl}
P \equiv 2 & \mathscr{B} x_{1}^{2}-4 \mathfrak{B}^{\prime} x_{1} x_{2}-2\left(\mathfrak{B}_{v}+A^{\prime} \mathfrak{B}+\phi\right) x_{1} x_{3}+2 \mathfrak{B}\left(\mathfrak{B}_{v}-A^{\prime} \mathfrak{B}\right) x_{2}^{2} \\
& +2\left(2 \mathfrak{B}_{v} \mathfrak{P}^{\prime}+2 \mathfrak{B} \mathfrak{B}_{v}^{\prime}+2 A^{\prime} \mathfrak{B} \mathfrak{B}^{\prime}+\mathfrak{B} K-\mathfrak{A}^{\prime \prime} \mathfrak{B}^{2}\right) x_{2} x_{3} \\
& +\left(\phi_{v}+4 A^{\prime} \phi-4 \mathfrak{U}^{\prime \prime} \mathfrak{B} \mathfrak{B}^{\prime}+2 \mathfrak{B} \mathfrak{C}^{\prime \prime}\right) x_{3}^{2}
\end{array}
$$


If we allow $y$ to move along a curve $v=$ const., $\delta v=0$. The osculating conic of this curve remains unchanged if and only if

$$
3 \mathfrak{B} \phi_{u}-4 \mathfrak{B}_{u} \phi-6 \mathfrak{B} B^{\prime} \phi+6 \mathfrak{B}^{2} H=0 \text {. }
$$

We see, therefore, that (99) is the necessary and sufficient condition for a net whose curves $v=$ const. are conics. Similarly if

$$
3 \mathfrak{U}{ }^{\prime \prime} \psi_{v}-4 \mathfrak{U}_{v}^{\prime \prime} \psi-6 \mathfrak{U}^{\prime \prime} A^{\prime} \psi+6 \mathfrak{A}^{\prime 2} K=0
$$

the curves $u=$ const. will be conics.

In general we see that the osculating conics of the curves $v=$ const. in the vicinity of the point $(u, v)$ belong to the two-parameter family of conics

$$
\lambda x_{3}^{2}+\mu M+\pi P=0 .
$$

The points of intersection of the conics $M$ and $P$ are of special interest. If they are fixed, the conics which osculate the curves $v=$ const. at the points in which they meet a fixed curve $u=$ const., will form a pencil, but we shall not attempt at present to deduce the analytical conditions for this special case.

We may of course obtain a formula similar to (97) for all of the conics which osculate the curves $u=$ const. in the vicinity of a given point $(u, v)$. It is clear that these two systems of conics together with their Jacobians and their other covariants must give rise to a very large number of relations. The exhaustive discussion of these will not here be attempted. We are satisfied in so far as we have reduced the problem to a purely algebraic one.

Let

\section{§ 7. Differential equations of the net in line-coördinates.}

$$
y^{(k)}=f^{(k)}(u, v)
$$

be the finite equations of the net, the triangle of reference being arbitrary. The equations of the lines tangent to the curves $v=$ const. and $u=$ const. at the point $(u, v)$ will be

$$
\left|\begin{array}{lll}
x_{1} & x_{2} & x_{3} \\
y^{(1)} & y^{(2)} & y^{(3)} \\
y_{u}^{(1)} & y_{u}^{(2)} & y_{u}^{(3)}
\end{array}\right|=0 \quad \text { and } \quad\left|\begin{array}{lll}
x_{1} & x_{2} & x_{3} \\
y^{(1)} & y^{(2)} & y^{(3)} \\
y_{v}^{(1)} & y_{v}^{(2)} & y_{v}^{(3)}
\end{array}\right|=0
$$

respectively, or

$$
Y^{(1)} x_{1}+Y^{(2)} x_{2}+Y^{(3)} x_{3}=0 \quad \text { and } \quad Z^{(1)} x_{1}+Z^{(2)} x_{2}+Z^{(3)} x_{3}=0,
$$

where $Y^{(k)}$ and $Z^{(k)}$, the coördinates of these two lines, are binary determinants of the types

$$
Y=\left|y, y_{u}\right|, \quad Z=\left|y, y_{v}\right|
$$


respectively. We propose to find the systems of partial differential equations determined by $Y^{(1)}, Y^{(2)}, Y^{(3)}$ and $Z^{(1)}, Z^{(2)}, Z^{(3)}$ respectively.

We find by differentiation

where

$$
\begin{aligned}
& Y_{u}=a Y+b Z, \\
& Y_{v}=a^{\prime} Y+b^{\prime} Z-W, \\
& Z_{u}=a^{\prime} Y+b^{\prime} Z+W, \\
& Z_{v}=a^{\prime \prime} Y+b^{\prime \prime} Z,
\end{aligned}
$$

We find further

$$
W=\left|y_{u}, y_{v}\right|
$$

$$
\begin{aligned}
& W_{u}=\left(a+b^{\prime}\right) W-c^{\prime} Y+c Z, \\
& W_{\bullet}=\left(a^{\prime}+b^{\prime \prime}\right) W-c^{\prime \prime} Y+c^{\prime} Z .
\end{aligned}
$$

Consequently we shall have

$$
\begin{aligned}
Y_{u u} & =\left(a_{u}+a^{2}+a^{\prime} b\right) Y+\left(b_{u}+a b+b b^{\prime}\right) Z+b W, \\
Y_{u v} & =\left(a_{v}+a a^{\prime}+a^{\prime \prime} b\right) Y+\left(b_{v}+a b^{\prime}+b b^{\prime \prime}\right) Z-a W \\
& =\left(a_{u}^{\prime}+a a^{\prime}+a^{\prime} b^{\prime}+c^{\prime}\right) Y+\left(b_{u}^{\prime}+a^{\prime} b+b^{\prime 2}-c\right) Z-a W, \\
Y_{v v} & =\left(a_{v}^{\prime}+a^{\prime 2}+a^{\prime \prime} b^{\prime}+c^{\prime \prime}\right) Y+\left(b_{o}^{\prime}+a^{\prime} b^{\prime}+b^{\prime} b^{\prime \prime}-c^{\prime}\right) Z-\left(2 a^{\prime}+b^{\prime \prime}\right) W,
\end{aligned}
$$

the two values of $Y_{u v}$ being identical on account of (5), and

$$
\begin{aligned}
Z_{u u} & =\left(a_{u}^{\prime}+a^{\prime 2}+a^{\prime} b^{\prime}-c^{\prime}\right) Y+\left(b_{u}^{\prime}+b^{\prime 2}+a^{\prime} b+c\right) Z+\left(2 a+b^{\prime}\right) W, \\
Z_{u v} & =\left(a_{v}^{\prime}+a^{\prime 2}+a^{\prime \prime} b^{\prime}-c^{\prime \prime}\right) Y+\left(b_{v}^{\prime}+a^{\prime} b^{\prime}+b^{\prime} b^{\prime \prime}+c^{\prime}\right) Z+b^{\prime \prime} W \\
& =\left(a_{u}^{\prime \prime}+a a^{\prime \prime}+a^{\prime} b^{\prime \prime}\right) Y+\left(b_{u}^{\prime \prime}+a^{\prime \prime} b+b^{\prime} b^{\prime \prime}\right) Z+b^{\prime \prime} W \\
Z_{v v} & =\left(a_{v}^{\prime \prime}+a^{\prime} a^{\prime \prime}+a^{\prime \prime} b^{\prime \prime}\right) Y+\left(b_{v}^{\prime \prime}+a^{\prime \prime} b^{\prime}+b^{\prime \prime 2}\right) Z-a^{\prime \prime} W .
\end{aligned}
$$

From (103) we have

$$
\begin{aligned}
b Z & =-a Y+Y_{u}, \quad a^{\prime \prime} Y=-b^{\prime \prime} Z+Z_{v}, \\
b W & =\left(a^{\prime} b-a b^{\prime}\right) Y+b^{\prime} Y_{u}-b Y_{v}, \\
a^{\prime \prime} W & =\left(a^{\prime} b^{\prime \prime}-a^{\prime \prime} b^{\prime}\right) Z+a^{\prime \prime} Z_{u}-a^{\prime} Z_{v},
\end{aligned}
$$

which values substituted in (106) and (107) will give the desired two systems of partial differential equations. We find in this way :

$$
Y_{u u}=\left(a+2 b^{\prime}+\frac{b_{u}}{b}\right) Y_{u}-b Y_{\mathrm{o}}+\left(a_{u}-a \frac{b_{u}}{b}+2 a^{\prime} b-2 a b^{\prime}\right) Y,
$$




$$
\begin{aligned}
Y_{u v}= & \left(b^{\prime \prime}+\frac{b_{v}}{b}\right) Y_{u}+a Y_{v}+\left(a_{v}-a \frac{b_{v}}{b}+a^{\prime \prime} b-a b^{\prime \prime}\right) Y, \\
Y_{v v}= & \frac{1}{b}\left(b_{v}^{\prime}-c^{\prime}-a^{\prime} b^{\prime}\right) Y_{u}+\left(2 a^{\prime}+b^{\prime \prime}\right) Y_{v} \\
& +\frac{1}{b}\left[b a_{v}^{\prime}-a b_{v}^{\prime}+b^{\prime}\left(a^{\prime \prime} b-a b^{\prime \prime}\right)+b c^{\prime \prime}+a c^{\prime}+\left(a^{\prime}+b^{\prime \prime}\right)\left(a b^{\prime}-a^{\prime} b\right)\right] Y .
\end{aligned}
$$

If we assume that the system (4) is written in its canonical form, we may write more simply

$$
\begin{aligned}
Y_{u u} & =\left(B^{\prime}+\frac{B_{u}}{B}\right) Y_{u}-B Y_{v}+\left(-B_{u}^{\prime}+B^{\prime} \frac{B_{u}}{B}+2 A^{\prime} B+2 B^{\prime 2}\right) Y, \\
\text { (109) } Y_{u v} & =\left(-A^{\prime}+\frac{B_{v}}{B}\right) Y_{u}-B^{\prime} Y_{v}+\left(-B_{v}^{\prime}+B^{\prime} \frac{B_{v}}{B}+A^{\prime \prime} B-A^{\prime} B^{\prime}\right) Y, \\
Y_{v v} & =-\frac{K}{B} Y_{u}+A^{\prime} Y_{v}+\left(C^{\prime \prime}+A_{v}^{\prime}+A^{\prime \prime} B^{\prime}+\frac{B^{\prime} K}{B}\right) Y,
\end{aligned}
$$

and similarly

$$
\begin{aligned}
& Z_{u u}=B^{\prime} Z_{u}-\frac{H}{A^{\prime \prime}} Z_{v}+\left(C+B_{u}^{\prime}+A^{\prime} B-\frac{A^{\prime} H}{A^{\prime \prime}}\right) Z \\
& Z_{u v}=-A^{\prime} Z_{u}+\left(-B^{\prime}+\frac{A_{u}^{\prime \prime}}{A^{\prime \prime}}\right) Z_{v}+\left(-A_{u}^{\prime}+A^{\prime} \frac{A_{u}^{\prime \prime}}{A^{\prime \prime}}+A^{\prime \prime} B-A^{\prime} B^{\prime}\right) Z \\
& Z_{v v}=-A^{\prime \prime} Z_{u}+\left(A^{\prime}+\frac{A_{v}^{\prime \prime}}{A^{\prime \prime}}\right) Z_{v}+\left(-A_{v}^{\prime}+A^{\prime} \frac{A_{v}^{\prime \prime}}{A^{\prime \prime}}+2 A^{\prime \prime} B^{\prime}+2 A^{\prime 2}\right) Z
\end{aligned}
$$

where, of course, $A^{\prime \prime}$ and $B$ are assumed to be different from zero.

Neither of these systems is in its canonical form. According to the developments of section 2 we may reduce them to their canonical form by the substitutions

$$
Y=\sqrt[3]{B} \bar{Y}, \quad Z=\sqrt[3]{A^{\prime \prime}} \bar{Z}
$$

Let us denote the coefficients of the new systems, i. e., the seminvariants of $(109)$ and $(110)$ by $\alpha^{(u)}, \beta^{(u)}, \gamma^{(u)}, \cdots \gamma^{(u)^{\prime \prime}}$ and $\alpha^{(v)} \cdots \gamma^{(v)^{\prime \prime}}$ respectively. Then we shall have

$$
\begin{array}{llrl}
\alpha^{(u)}=B^{\prime}+\frac{1}{3} \frac{B_{u}}{B}, & \alpha^{(u)^{\prime}}=-A^{\prime}+\frac{2}{3} \frac{B_{v}}{B}, & \alpha^{(u)^{\prime \prime}}=-\frac{K}{B}, \\
\beta^{(u)}=-B, & \beta^{(u)^{\prime}}=-B^{\prime}-\frac{1}{3} \frac{B_{u}}{B}, & \beta^{(u)^{\prime \prime}}=A^{\prime}-\frac{2}{3} \frac{B_{v}}{B},
\end{array}
$$

(112) $\gamma^{(u)}=2 A^{\prime} B+2 B^{\prime 2}+\frac{4}{3} B^{\prime} \frac{B_{u}}{B}-B_{u}^{\prime}-\frac{1}{3} B_{v}+\frac{5}{9} \frac{B_{u}^{2}}{B^{2}}-\frac{1}{3} \frac{B_{u u}}{B^{-}}$, 


$$
\begin{aligned}
& \gamma^{(u)^{\prime}}=A^{\prime \prime} B-A^{\prime} B^{\prime}-\frac{1}{3} A^{\prime} \frac{B_{u}}{B}+\frac{2 B^{\prime}}{3} \frac{B_{v}}{B}-B_{v}^{\prime}+\frac{5}{9} \frac{B_{u} B_{v}}{B^{2}}-\frac{1}{3} \frac{B_{u v}}{B} \\
& \gamma^{(u)^{\prime \prime}}=C^{\prime \prime}+A^{\prime \prime} B^{\prime}+\frac{B^{\prime} K}{B}+\frac{1}{3} A^{\prime} \frac{B_{v}}{B}-\frac{1}{3} \frac{K}{B} \frac{B_{u}}{B}+A_{v}^{\prime}+\frac{2}{9} \frac{B_{v}^{2}}{B^{2}}-\frac{1}{3} \frac{B_{v v}}{B}
\end{aligned}
$$

and

$$
\begin{aligned}
\alpha^{(v)} & =B^{\prime}-\frac{2}{3} \frac{A_{u}^{\prime \prime}}{A^{\prime \prime}}, \quad \alpha^{(v)^{\prime}}=-A^{\prime}-\frac{1}{3} \frac{A_{v}^{\prime \prime}}{A^{\prime \prime}}, \quad \alpha^{(v)^{\prime \prime}}=-A^{\prime \prime}, \\
\beta^{(v)} & =-\frac{H}{A^{\prime \prime}}, \quad \beta^{(v)^{\prime}}=-B^{\prime}+\frac{2}{3} \frac{A_{u}^{\prime \prime}}{A^{\prime \prime}}, \quad \beta^{(v)^{\prime \prime}}=A^{\prime}+\frac{1}{3} \frac{A_{v}^{\prime \prime}}{A^{\prime \prime}}, \\
\gamma^{(v)} & =C+A^{\prime} B-\frac{A^{\prime} H}{A^{\prime \prime}}+\frac{1}{3} B^{\prime} \frac{A_{u}^{\prime \prime}}{A^{\prime \prime}}-\frac{1}{3} \frac{H}{A^{\prime \prime}} \frac{A_{v}^{\prime \prime}}{A^{\prime \prime}}+B_{u}^{\prime}+\frac{2}{9}\left(\frac{A_{u}^{\prime \prime}}{A^{\prime \prime}}\right)^{2}-\frac{1}{3} \frac{A_{u u}^{\prime \prime}}{A^{\prime \prime}}, \\
\gamma^{(v)^{\prime}} & =A^{\prime \prime} B-A^{\prime} B^{\prime}+\frac{2}{3} A^{\prime} \frac{A_{u}^{\prime \prime}}{A^{\prime \prime}}-\frac{1}{3} B^{\prime} \frac{A_{v}^{\prime \prime}}{A^{\prime \prime}}-A_{u}^{\prime}+\frac{5}{9} \frac{A_{u}^{\prime \prime}}{\left(A_{v}^{\prime \prime}\right)^{\prime \prime}}-\frac{1}{3} \frac{A_{u v}^{\prime \prime}}{A^{\prime \prime}}, \\
\gamma^{(v)} & =2 A^{\prime \prime} B^{\prime}+2\left(A^{\prime}\right)^{2}+\frac{4}{3} A^{\prime} \frac{A_{v}^{\prime \prime}}{A^{\prime \prime}}-\frac{1}{3} A_{u}^{\prime \prime}-A_{v}^{\prime}+\frac{5}{9}\left(\frac{A_{v}^{\prime \prime}}{A^{\prime \prime}}\right)^{2}-\frac{1}{3} \frac{A_{v v}^{\prime \prime}}{A^{\prime \prime}}
\end{aligned}
$$

These equations will obviously be of service whenever we wish to characterize a net by properties of the tangents of its curves. By means of (109) the lines of the plane are assembled in two different ways; those for which $v$ has a constant value $c$ are tangent to the curve $v=c$ of our original net; those for which $u$ has a constant value are tangent to a curve of the first Laplacian transformed net. By means of equations (110) the lines of the plane are grouped in a similar way with reference to the curves $u=$ const. of the original net and the minus first Laplacian transformed net.

In this whole theory we might, of course, adopt a dual interpretation. Instead of a net we should then have two one-parameter families of curves so related that, for every line of a certain domain, there exists one curve of each family tangent to it. We have seen instances of such a relation in the theory of the Laplacian transformation.

The University of Chicago, December 12, 1910. 